\title{
Pacific
}

Journal of

Mathematics

\section{REALIZING PROFINITE REDUCED SPECIAL GROUPS}

Vincent ASTIER AND Hugo Mariano 


\title{
REALIZING PROFINITE REDUCED SPECIAL GROUPS
}

\author{
Vincent Astier AND Hugo Mariano
}

\begin{abstract}
Special groups are an axiomatization of the algebraic theory of quadratic forms over fields. It is known that any finite reduced special group is the special group of some field. We show that any special group that is the projective limit of a projective system of finite reduced special groups is also the special group of some field.
\end{abstract}

\section{Introduction}

The theory of special groups is an axiomatization of the algebraic theory of quadratic forms, introduced in [Dickmann and Miraglia 2000]. The class of special groups, together with its morphisms, forms a category. As for other such axiomatizations, the main examples of special groups are provided by fields, in this case by applying the special group functor, which associates to each field $F$ a special group $G(F)$ describing the theory of quadratic forms over $F$.

The category of special groups is equivalent to that of abstract Witt rings via covariant functors, while the category of reduced special groups is equivalent, via the restriction of the same covariant functors, to the category of reduced abstract Witt rings (see [Dickmann and Miraglia 2000, 1.25 and 1.26]; recall that the special group of a field $F$ is reduced if and only if $F$ is formally real and Pythagorean). The category of reduced special groups is also equivalent, via contravariant functors, to the category of abstract spaces of orderings; see Chapter 3 of the same reference.

The question whether it is possible to realize every (reduced) special group as the special group of some (formally real, Pythagorean) field is still open, but the case of finite reduced special groups (actually of reduced special groups of finite chain length) has been positively answered by the combination of two results: Kula [1979], building on techniques introduced in [Bröcker 1977] for the field case, showed that the product of two finite special groups of (formally real, Pythagorean) fields is still the special group of some (formally real, Pythagorean) field; then Marshall [1980] showed that every finite reduced special group can be constructed from the special group of any real closed field by applying a finite number of times the operations of product and extension. (Marshall's result is actually stated and

MSC2000: 03C65, 11E81.

Keywords: quadratic forms, special groups, projective limit, profinite, representation theorems. 
proved for abstract spaces of orderings.) Since the extension of the special group of a (formally real, Pythagorean) field is still the special group of a (formally real, Pythagorean) field, it shows that every finite reduced special group (or reduced special group of finite chain length) is realized as the special group of a field.

After finite reduced special groups, the simplest objects to consider are probably projective limits of finite reduced special groups, that is, profinite reduced special groups. They have already been studied, for example, in [Astier and Tressl 2005; Lira de Lima 1997; Mariano 2003], and notably in [Kula et al. 1984], where the question of the realization of these special groups by fields is considered and where it is shown (as Corollary 4.7) that every profinite reduced special group is isomorphic to a quotient of the reduced special group of some field.

In this paper, we improve on this result by showing that every profinite reduced special group is isomorphic to the special group of some (necessarily formally real and Pythagorean) field.

\section{Preliminaries}

Definition 2.1. Let $A, B, A^{\prime}, B^{\prime}$ be objects in a category $\mathscr{C}$, and let $\lambda: A \rightarrow B$, $\lambda^{\prime}: A^{\prime} \rightarrow B^{\prime}$ be $\mathscr{C}$-morphisms. Then $\lambda, \lambda^{\prime}$ are said to be naturally identified (in symbols, $\lambda \cong \lambda^{\prime}$ ) if and only if there are $\mathscr{C}$-isomorphisms $i_{A}: A \rightarrow A^{\prime}, i_{B}: B \rightarrow B^{\prime}$ such that the following diagram

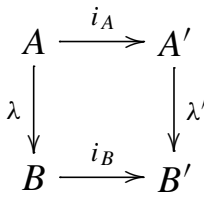

commutes. In this case, we also say that $\lambda$ and $\lambda^{\prime}$ are naturally identified via $i_{A}, i_{B}$.

On special groups. We assume some familiarity with the theory of special groups, as presented in [Dickmann and Miraglia 2000], and only introduce the following notation:

If $G$ is a special group, $\operatorname{Ssat}(G)$ denotes the poset of saturated subgroups of $G$, ordered by inclusion. We recall that if $\Delta \in \operatorname{Ssat}(G)$, then $G / \Delta$ is a reduced special group if and only if $\Delta \subsetneq G$, if and only if $-1 \notin \Delta$.

Definition 2.2. A profinite reduced special group is the projective limit of a projective system of finite reduced special groups.

If $\left(G_{i}^{\prime}, f_{i j}^{\prime}\right)_{i \leq j \in I}$ is a projective system of finite reduced special groups, where $(I, \leq)$ is a downward directed poset, and if $G$ is the projective limit of this system, the fact that $G$ is indeed a special group (with the structure induced by its inclusion in the product $\prod_{i \in I} G_{i}^{\prime}$ ) follows immediately from [Dickmann and Miraglia 2003, 
Theorem 3.24]. Moreover, as proved in [Lira de Lima 1997, Proposition 1.9.11], it is always possible to describe $G$ as the projective limit of a projective system $\left(G_{i}, f_{i j}\right)_{i \leq j \in I}$ having the following properties:

(1) For every $i \in I, G_{i}$ is $G / \Delta_{i}$ with $\Delta_{i}$ saturated subgroup of $G$ of finite index;

(2) For every $i \leq j \in I, \Delta_{i} \subseteq \Delta_{j}$ and $f_{i j}$ is the canonical projection of special groups induced by this inclusion.

We briefly sketch the argument: Let $\iota: G \hookrightarrow \prod_{j \in I} G_{j}^{\prime}$ be the canonical embedding given by the definition of projective limit, and let $\pi_{i}: \prod_{j \in I} G_{j}^{\prime} \rightarrow G_{i}^{\prime}$ be the canonical projection. We define $\Delta_{i}:=\operatorname{ker}\left(\pi_{i} \circ \iota\right), G_{i}:=G / \Delta_{i}$ and, for $i \leq j \in I, f_{i j}$ to be the canonical projection induced by $\Delta_{i} \subseteq \Delta_{j}$. The system $\left(G_{i}, f_{i j}\right)_{i \leq j \in I}$ is a projective system, whose projective limit is isomorphic to $G$, via the map $g \in G \mapsto\left(g . \Delta_{i}\right)_{i \in I} \in \lim \left(G / \Delta_{i}, f_{i j}\right)_{i \leq j \in I}$.

Remark 2.3. If $\mu=\left(M_{i}, f_{i j}\right)_{i \leq j \in I}$ is any projective system, and if $i^{\prime} \in I$, by restricting this system to the set $I^{\prime}:=\left\{i \in I \mid i \leq i^{\prime}\right\}$ we obtain a new system

$$
\mathcal{M}^{\prime}:=\left(M_{i}, f_{i j}\right)_{i \leq j \in I^{\prime}}
$$

Since $I^{\prime}$ is coinitial in $I, \mathcal{M}$ and $\mathcal{M}^{\prime}$ have isomorphic projective limits, and $\mathcal{M}^{\prime}$ possesses the following extra property:

(3) The index set of the projective system has a maximum element.

Definition 2.4. We call adequate a projective system of special groups that satisfies conditions (1), (2) and (3) above.

We will adhere to the following convention throughout this paper: Let $(I, \leq)$ be a downward directed poset. If $(I, \leq)$ has a maximum element, we will denote it by $\top$, and if $(I, \leq)$ has a minimum element (which happens for instance if $I$ is finite), we will denote it by $\perp$.

Let $G_{0}, G_{1}$ be abstract groups and denote by $\pi_{0}: G_{0} \times G_{1} \rightarrow G_{0}:\left(g_{0}, g_{1}\right) \mapsto g_{0}$, $\pi_{1}: G_{0} \times G_{1} \rightarrow G_{1}:\left(g_{0}, g_{1}\right) \mapsto g_{1}$ the canonical projections and by $\iota_{0}: G_{0} \longmapsto$ $G_{0} \times G_{1}: g_{0} \mapsto\left(g_{0}, 1\right), \iota_{1}: G_{1} \longmapsto G_{0} \times G_{1}: g_{1} \mapsto\left(1, g_{1}\right)$ the canonical injections.

The statements in the next paragraph are straightforward.

Fact 2.5. Let $G_{0}, G_{1}$ be special groups. Then the canonical map

$$
\begin{aligned}
\psi: \operatorname{Ssat}\left(G_{0} \times G_{1}\right) & \rightarrow \operatorname{Ssat}\left(G_{0}\right) \times \operatorname{Ssat}\left(G_{1}\right) \\
\Delta & \mapsto\left(\iota_{0}^{-1}[\Delta], \iota_{1}^{-1}[\Delta]\right)=\left(\pi_{0}[\Delta], \pi_{1}[\Delta]\right)
\end{aligned}
$$

is an order-preserving bijection, whose inverse is $\left(\Delta_{0}, \Delta_{1}\right) \stackrel{\psi^{-1}}{\mapsto} \Delta_{0} \times \Delta_{1}$. In particular, if $\Delta \in \operatorname{Ssat}\left(G_{0} \times G_{1}\right)$ and $\left(\Delta_{0}, \Delta_{1}\right):=\left(\iota_{0}^{-1}[\Delta], \iota_{1}^{-1}[\Delta]\right)$, then $\Delta=\Delta_{0} \times \Delta_{1}$ and $\Delta$ is proper if and only if $\Delta_{0}$ or $\Delta_{1}$ is proper. Moreover: 
- The canonical surjective morphism of special groups

$$
G_{0} \times G_{1} \rightarrow G_{0} / \Delta_{0} \times G_{1} / \Delta_{1}
$$

induces a natural isomorphism of special groups ${ }^{1}$

$$
\bar{q}_{\Delta}:\left(G_{0} \times G_{1}\right) / \Delta \stackrel{\cong}{\cong} G_{0} / \Delta_{0} \times G_{1} / \Delta_{1} .
$$

- If $\Delta \subseteq \Delta^{\prime} \in \operatorname{Ssat}\left(G_{0} \times G_{1}\right)$, then the projection $\left(G_{0} \times G_{1}\right) / \Delta \rightarrow\left(G_{0} \times G_{1}\right) / \Delta^{\prime}$ is naturally identified, via the isomorphisms $\bar{q}_{\Delta}, \bar{q}_{\Delta^{\prime}}$, with the (product) projection $G_{0} / \Delta_{0} \times G_{1} / \Delta_{1} \rightarrow G_{0} / \Delta_{0}^{\prime} \times G_{1} / \Delta_{1}^{\prime}$.

On projective systems of (valued) fields. Let $(I, \leq)$ be a poset. For each $i, j \in I$ such that $i \leq j$ we define $d(i, j):=\max \{$ length of a chain from $i$ to $j\} \in \mathbb{N} \cup\{\infty\}$. If $i \not \leq j$ then we set $d(i, j):=-\infty$. Of course, if $i$ and $j$ are comparable, we have $d(i, j)=d(j, i)$ if and only if $i=j$, if and only if $d(i, j)=0$.

We will often consider $(I, \leq)$ as a directed graph whose vertices are the elements of $I$, and where there is an edge from $i$ to $j$ if and only if $i \leq j$ and $d(i, j)=1$.

We first remark that it is possible to describe some projective systems of fields as projective systems whose morphisms are all inclusions.

Remark 2.6. Let $\mathscr{F}:=\left(F_{i}, f_{i j}\right)_{i \leq j \in I}$ be a projective system of fields over a downward directed poset $(I, \leq)$ with maximum element $T \in I$. Then there is an isomorphic projective system of fields $\mathscr{F}^{\prime}=\left(F_{i}^{\prime}, \iota_{i j}\right)_{i \leq j \in I}$ such that, if $i \leq j \in I$, then $F_{i}^{\prime} \subseteq F_{j}^{\prime}$ and the morphism of fields $\iota_{i j}: F_{i}^{\prime} \rightarrow F_{j}^{\prime}$ is the inclusion. The projective limit of the system $\mathscr{F}$ is thus isomorphic to the intersection of the fields $F_{i}^{\prime}, i \in I$.

We briefly sketch the argument. For each $i \in I$, we define $F_{i}^{\prime}:=f_{i \top}\left[F_{i}\right] \subseteq F_{\top}$. Since for $i \leq j \in I, f_{i \top}=f_{j \top} \circ f_{i j}$, we obtain $F_{i}^{\prime} \subseteq F_{j}^{\prime}$, so we can define $\iota_{i j}$ to be this inclusion. It follows that $\mathscr{F}$ and $\mathscr{F}^{\prime}$ are isomorphic via the morphisms $\left(f_{i \top}\right)_{i \in I}$. Therefore: $\lim _{\longleftarrow}\left(F_{j}, f_{i j}\right)_{i \leq j \in I} \cong \lim _{\longleftarrow}\left(F_{j}^{\prime}, \iota_{i j}\right)_{i \leq j \in I} \cong \bigcap_{i \in I} F_{i}^{\prime} \subseteq F_{\top}^{\prime}$.

The next results lead to Corollary 2.10 , which shows that any finite projective system of fields of characteristic zero, whose index set has a maximum element, is isomorphic to the projective system given by the residues of a finite projective system of valued fields. We first fix some notation:

If $(K, v)$ is a valued field, we denote by $K v$ or by $\bar{K}$ (if there is no risk of confusion about which valuation we consider) the residue field of $v$, by $v K$ its value group, by $O_{K}$ the valuation ring associated to $v$ and by $M_{K}$ its maximal ideal (if there is no ambiguity about the valuation $v$ under consideration). If $a \in O_{K}$, we denote by $a v$ or $\bar{a}$ (once again if there is no risk of confusion) the class of $a$ in the residue field $\bar{K}$. Finally, if $v$ has rank one, $K^{v}$ denotes a completion of $K$ with respect to $v$.

\footnotetext{
${ }^{1}$ That are reduced if $\Delta$ is proper or, otherwise, the trivial special group $\{1\}$.
} 
If $\mathscr{F}=\left(F_{i}, \xi_{i j}\right)_{i \leq j \in I}$ is a projective system of fields, we denote by $G(\mathscr{F})$ the system $\left(G\left(F_{i}\right), G\left(\xi_{i j}\right)\right)_{i \leq j \in I}$ obtained from $\mathscr{F}$ by applying the special group functor $G$.

If $\mathscr{F}=\left(\left(F_{i}, v_{i}\right), \xi_{i j}\right)_{i \leq j \in I}$ is a projective system of valued fields, we denote by res $\mathscr{F}$ or $\mathscr{F} v$ the induced residue projective system $\left(F_{i} v_{i},(\xi v)_{i j}\right)_{i \leq j \in I}$, where the $(\xi v)_{i j}$ are the induced morphisms of fields.

If a projective system of fields or of valued fields is denoted by $\left(F_{i}\right)_{i \in I}$ or $\left(F_{i}, v_{i}\right)_{i \in I}$, without mention of the morphisms, it means that the morphisms are all inclusions (from a field within all fields with larger index).

Lemma 2.7. Let $(K, v)$ be a henselian valued field of residue characteristic zero and let $L$ be a subfield of $K$. Let $N$ be a subfield of $\bar{K}$ such that $\bar{L} \subseteq N \subseteq \bar{K}$. Then there is a field $M$ such that $L \subseteq M \subseteq K$ and $\bar{M}=N$. Moreover, if $[N: \bar{L}]$ is algebraic, respectively finite, then $M$ can be chosen such that $[M: L]$ is algebraic, respectively finite.

Proof. Write $N=\bar{L}(X)\left(\alpha_{i}, i \in \beta\right)$, where $X$ is a transcendence basis of $N$ over $\bar{L}$ and $\left(\alpha_{i}, i \in \beta\right)$ is a (possibly infinite) tuple of elements that are algebraic over $\bar{L}(X)$, indexed by an ordinal $\beta$. Let $Y$ be a set of transcendental elements over $L$ such that $\bar{Y}=X$. By [Engler and Prestel 2005, Corollary 2.2.2], the restriction of $v$ to $L(Y)$ is the Gauss extension of $v$ from $L$ to $L(Y)$. In particular, $\overline{L(Y)}=\bar{L}(X)$.

We now proceed by induction on $k \in \beta$ to find elements $a_{i} \in K, i<k$, such that $\overline{L(Y)\left(a_{i}, i<k\right)}=\bar{L}(X)\left(\alpha_{i}, i<k\right)$.

If $k=0$ there is nothing to prove since $\overline{L(Y)}=\bar{L}(X)$.

Assume we have found all $a_{i}$ for $i<k$. Let $N_{k}=L(Y)\left(a_{i}, i<k\right)$ and $M_{k}=$ $\bar{L}(X)\left(\alpha_{i}, i<k\right)$. By hypothesis we have $\bar{N}_{k}=M_{k}$. Let $P \in O_{K}[T]$ be a unitary polynomial such that $\bar{P}$ is the minimal polynomial of $\alpha_{k}$ over $\bar{L}(X)$. Let $a_{k}$ be a root of $P$ in $K$ such that $\bar{a}_{k}=\alpha_{k}$ (it exists since $(K, v)$ is henselian of residue characteristic zero). We have $\overline{N_{k}\left(a_{k}\right)} \supseteq M_{k}\left(\alpha_{k}\right)$ and the fundamental inequality [Engler and Prestel 2005, Theorem 3.3.4] tells us that

$$
\left[\overline{N_{k}\left(a_{k}\right)}: \overline{N_{k}}\right] \leq\left[N_{k}\left(a_{k}\right): N_{k}\right](\leq \operatorname{deg} P) .
$$

Since $\left[M_{k}\left(\alpha_{k}\right): M_{k}\right]=\operatorname{deg} P$, it follows that $\overline{N_{k}\left(a_{k}\right)}=M_{k}\left(\alpha_{k}\right)$, which is the desired result.

Definition 2.8. Let $(K, v)$ be a valued field and let $\left(E_{i}\right)_{i<n}$ and $\left(F_{i}\right)_{i<n}$ be two sequences of fields of the same length $n$. We say that $\left(F_{i}\right)_{i<n}$ is a good residue of $\left(E_{i}\right)_{i<n}$ in $(K, v)$ if

(1) $E_{i} \subseteq K$ and $F_{i} \subseteq \bar{K}$ for $i<n$;

(2) For every $A \subseteq\{0, \ldots, n-1\}, \overline{\left\langle E_{i}, i \in A\right\rangle}=\left\langle F_{i}, i \in A\right\rangle$ (where $\left\langle L_{i}, i \in A\right\rangle$ denotes the compositum of the fields $L_{i}$ ). 
Lemma 2.9. Let $(K, v)$ be a henselian valued field of residue characteristic zero, and let $\left(E_{i}\right)_{i<n}$ and $\left(F_{i}\right)_{i<n}$ be two sequences of fields of length $n$ such that $\left(F_{i}\right)_{i<n}$ is a good residue of $\left(E_{i}\right)_{i<n}$ in $(K, v)$. Let $\left(F_{i}^{\prime}\right)_{i<m}$ be a sequence of subfields of $\bar{K}$, and let, for $i \in\{0, \ldots m-1\}$

$$
A_{i}=\left\{j \in\{0, \ldots, n-1\} \mid F_{j} \subseteq F_{i}^{\prime}\right\} .
$$

Then there is a sequence $\left(E_{i}^{\prime}\right)_{i<m}$ of subfields of $K$ such that

(1) for every $i \in\{0, \ldots, m-1\}$ and every $j \in A_{i}, E_{j} \subseteq E_{i}^{\prime}$ and $\operatorname{trdeg} E_{i}^{\prime} \mid E_{j}=$ $\operatorname{trdeg} F_{i}^{\prime} \mid F_{j}$

(2) $\left(F_{i}^{\prime}\right)_{i<m}$ is a good residue of $\left(E_{i}^{\prime}\right)_{i<m}$.

Proof. We will use the following reformulation of Remark 4.1.2(3) in [Engler and Prestel 2005]:

Fact 1. Let $(N, w)$ be a valued field and let $P, Q \in O_{N}[T]$ and $R \in N[T]$ be such that $P=Q R$. Assume that $Q$ is primitive (that is, $w^{\prime}(Q)=0$, where $w^{\prime}$ is the Gauss extension of $w$ to $N[T]$, i.e., $\min _{i \leq k} w\left(a_{i}\right)=0$ if one writes $\left.Q=a_{0}+\cdots+a_{k} T^{k}\right)$. Then $R \in O_{N}[T]$.

Proof. Write $P=a P_{1}$ and $R=c R_{1}$ with $a, c \in N$ and $P_{1}, R_{1} \in N[T]$ such that $w^{\prime}\left(P_{1}\right)=w^{\prime}\left(R_{1}\right)=0$ (so $\left.P_{1}, R_{1} \in O_{N}[T]\right)$. Then $w(c)=w^{\prime}(Q)+w^{\prime}\left(c R_{1}\right)=$ $w^{\prime}\left(Q c R_{1}\right)=w^{\prime}(Q R)=w^{\prime}(P) \geq 0$ since $P \in O_{N}[T]$. This yields $R=c R_{1}$ with $w^{\prime}(R)=w(c)+w^{\prime}\left(R_{1}\right)=w(c) \geq 0$, i.e., $R \in O_{N}[T]$.

We next fix some notation. For $A \subseteq\{0, \ldots, n-1\}$ we denote by $F_{A}$ the field $\left\langle F_{i}, i \in A\right\rangle$ and similarly by $E_{A}$ the field $\left\langle E_{i}, i \in A\right\rangle$.

For $i<m$ let $X_{i}=\left\{x_{i 1}, \ldots, x_{i k_{i}}\right\}$ be a transcendence basis of $F_{i}^{\prime}$ over $F_{A_{i}}=\bar{E}_{A_{i}}$, and let $Y_{i}=\left\{y_{i 1}, \ldots, y_{i k_{i}}\right\} \subseteq K$ be a set of transcendental elements over $E_{A_{i}}$ such that $\bar{Y}_{i}=X_{i}$. Note that by [Engler and Prestel 2005, corollary 2.2.2], it implies that the restriction of $v$ to $E_{A_{i}}\left(Y_{i}\right)$ is the Gauss extension of $v$ from $E_{A_{i}}$ to $E_{A_{i}}\left(Y_{i}\right)$. In particular we have $\overline{E_{A_{i}}\left(Y_{i}\right)}=\overline{E_{A_{i}}}\left(X_{i}\right)=F_{A_{i}}\left(X_{i}\right)$ (the last equality holds because $\left(F_{i}\right)_{i<n}$ is a good residue of $\left.\left(E_{i}\right)_{i<n}\right)$.

Write $F_{i}^{\prime}=F_{A_{i}}\left(X_{i}\right)\left(\alpha_{i}\right)$, where $\alpha_{i}=\left(\alpha_{i j}\right)_{j \in \beta_{i}}$ is a (possibly infinite) tuple of elements algebraic over $F_{A_{i}}\left(X_{i}\right)$. For $i<m$ and $j \in \beta_{i}$ let $P_{i j} \in O_{E_{A_{i}}\left(Y_{i}\right)}[T]$ be a unitary polynomial such that $\bar{P}_{i j}$ is the minimal polynomial of $\alpha_{i j}$ over $\overline{E_{A_{i}}\left(Y_{i}\right)}=$ $F_{A_{i}}\left(X_{i}\right)$, and let $a_{i j} \in O_{K}$ be a root of $P_{i j}$ with $\bar{a}_{i j}=\alpha_{i j}\left(a_{i j}\right.$ exists since $(K, v)$ is henselian of residue characteristic zero). We take for $E_{i}^{\prime}$ the field $E_{A_{i}}\left(Y_{i}\right)\left(a_{i}\right)$, where $a_{i}=\left(a_{i j}\right)_{j \in \beta_{i}}$. The first conclusion of the lemma is obviously satisfied. Let $A \subseteq\{0, \ldots, m-1\}$.

Claim. Let $L$ be a subfield of $K$ such that $(L, v)$ is henselian, $\left\langle F_{i}^{\prime}, i \in A\right\rangle \subseteq \bar{L}$, and $\left\langle E_{j}, j \in A_{i}, i \in A\right\rangle\left(Y_{i}, i \in A\right) \subseteq L$. Then $a_{i} \in L$ for every $i \in A$, i.e., $L \supseteq\left\langle E_{i}^{\prime}, i \in A\right\rangle$. 
Proof. Let $i \in A$ and $j \in \beta_{i}$. Since $\alpha_{i j} \in \bar{L}$ and $(L, v)$ is henselian (of residue characteristic zero), there is $b_{i j} \in O_{L}$ such that $\bar{b}_{i j}=\alpha_{i j}$ and $b_{i j}$ is a root of $P_{i j}$. Assume $b_{i j} \neq a_{i j}$. Then we can write $P_{i j}(T)=\left(T-a_{i j}\right)\left(T-b_{i j}\right) R(T)$ in $E_{A_{i}}\left(Y_{i}, a_{i j}, b_{i j}\right)$. But $P_{i j},\left(T-a_{i j}\right),\left(T-b_{i j}\right)$ each lie in $O_{E_{A_{i}}\left(Y_{i}, a_{i j}, b_{i j}\right)}[T]$ and $\left(T-a_{i j}\right)\left(T-b_{i j}\right)$ is primitive, so by Fact 1 we have $R(T) \in O_{E_{A_{i}}\left(Y_{i}, a_{i j}, b_{i j}\right)}[T]$. Going to the residue field $\bar{K}$ we get $\bar{P}_{i j}(T)=\left(T-\alpha_{i j}\right)^{2} \bar{R}(T)$, so $\alpha_{i j}$ is root of order at least 2 of $\bar{P}_{i j}$, which is impossible since $\bar{P}_{i j}$ is the minimal polynomial of $\alpha_{i j}$ and char $\bar{K}=0$. So $a_{i j}=b_{i j} \in L$. End of proof of the claim.

We have $E_{A}^{\prime}=\left\langle E_{i}^{\prime}, i \in A\right\rangle=\left\langle\left\langle E_{j}, j \in A_{i}\right\rangle\left(Y_{i}\right)\left(a_{i}\right), i \in A\right\rangle=\left\langle E_{j}, j \in A_{i}, i \in\right.$ $A\rangle\left(Y_{i}, i \in A\right)\left(a_{i}, i \in A\right)$, and

$$
\begin{aligned}
\overline{\left\langle E_{j}, j \in A_{i}, i \in A\right\rangle\left(Y_{i}, i \in A\right)} & =\overline{\left\langle E_{j}, j \in A_{i}, i \in A\right\rangle}\left(X_{i}, i \in A\right) \\
& =\left\langle F_{j}, j \in A_{i}, i \in A\right\rangle\left(X_{i}, i \in A\right) .
\end{aligned}
$$

Moreover,

$\left\langle F_{i}^{\prime}, i \in A\right\rangle=\left\langle\left\langle F_{j}, j \in A_{i}\right\rangle\left(X_{i}\right)\left(\alpha_{i}\right), i \in A\right\rangle=\left\langle F_{j}, j \in A_{i}, i \in A\right\rangle\left(X_{i}, i \in A\right)\left(\alpha_{i}, i \in A\right)$.

So $\left\langle F_{i}^{\prime}, i \in A\right\rangle$ is an algebraic extension of $\overline{\left\langle E_{j}, j \in A_{i}, i \in A\right\rangle\left(Y_{i}, i \in A\right)}$. In particular (see Lemma 2.7) there is an algebraic extension $E^{\prime \prime}$ of

$$
\left\langle E_{j}, j \in A_{i}, i \in A\right\rangle\left(Y_{i}, i \in A\right)
$$

(inside $K$ ) such that $\bar{E}^{\prime \prime}=\left\langle F_{i}^{\prime}, i \in A\right\rangle$. Let $\tilde{E}$ be the henselian closure of $E^{\prime \prime}$ in $(K, v)$. We have $\overline{\tilde{E}}=\left\langle F_{i}^{\prime}, i \in A\right\rangle, E^{\prime \prime} \subseteq \tilde{E}$. By the claim, since $\tilde{E}$ is henselian and $\tilde{\tilde{E}} \supseteq\left\langle F_{i}^{\prime}, i \in A\right\rangle$, we have $a_{i} \in \tilde{E}$ for every $i \in A$. It implies $E_{A}^{\prime} \subseteq \tilde{E}$, which gives, taking residues $\bar{E}_{A}^{\prime} \subseteq \overline{\tilde{E}}=\left\langle F_{i}^{\prime}, i \in A\right\rangle$. But by construction of the $E_{i}^{\prime}$ we obviously have $\bar{E}_{A}^{\prime} \supseteq\left\langle F_{i}^{\prime}, i \in A\right\rangle$. It follows that $\bar{E}_{A}^{\prime}=\left\langle F_{i}^{\prime}, i \in A\right\rangle$.

Corollary 2.10. Let $\mathscr{F}=\left(F_{i}\right)_{i \in I}$ be a finite projective system of fields of characteristic zero and let $\perp$ be the minimum of $I$. Assume that $(I, \leq)$ has a maximum $\top$ and let $\left(E_{\perp}, v_{\perp}\right)$ be a valued field such that $E_{\perp} v_{\perp} \cong F_{\perp}$. Then there is a projective system of valued fields $\left(E_{i}, v_{i}\right)_{i \in I}$ such that $\left(F_{i}\right)_{i \in I} \cong \operatorname{res}\left(E_{i}, v_{i}\right)_{i \in I}$ and, for every $i \in I, \operatorname{trdeg} E_{i}\left|E_{\perp}=\operatorname{trdeg} F_{i}\right| F_{\perp}$. Moreover:

- We can assume that all $\left(E_{i}, v_{i}\right), i \in I$, are henselian.

- If $v_{\perp}$ has rank one, then we can choose the valuations $v_{i}, i \in I$, such that they all have rank one.

Proof. We first show that there is a projective system of fields $\mathscr{F}^{\prime}=\left(F_{i}^{\prime}\right)_{i \in I}$ with $\mathscr{F}^{\prime} \cong \mathscr{F}$ and there is an extension $(K, v)$ of $\left(E_{\perp}, v_{\perp}\right)$ such that $\bar{K}=F_{\top}^{\prime}$, and such that $v$ has rank one if $v_{\perp}$ has rank one. In particular $\bar{K} \supseteq F_{i}^{\prime}$ for every $i \in I$.

Indeed, write $F_{\top}=F_{\perp}(X)(\bar{a})$, where $X$ is a set of elements transcendental over $F_{\perp}$ and $\bar{a}$ is a sequence of elements algebraic over $F_{\perp}(X)$. Take $Y$ a set of 
indeterminates with the same cardinality as $X$ and consider the Gauss extension $w$ of $v_{\perp}$ to $E_{\perp}(Y)$. Then $\overline{E_{\perp}(Y)} \cong F_{\perp}(X)$. Note that $w$ has rank one if $v_{\perp}$ has rank one. Using now for instance [Endler 1963, Satz 1], we find an (algebraic) extension $(K, v)$ of $\left(E_{\perp}(Y), w\right)$ such that $\bar{K}$ and $F_{\top}$ are isomorphic via a map which we denote by $h: \bar{K} \rightarrow F_{\top}$ (and with $v$ of rank one if $w$ has rank one). Define $F_{i}^{\prime}:=h^{-1}\left[F_{i}\right]$. This justifies the claim in the first paragraph of the proof.

To keep notation simple, we assume $\mathscr{F}=\mathscr{F}^{\prime}$ as above. We construct the valued fields $\left(E_{i}, v_{i}\right)$ (for $i \neq \perp$ ) as subfields of $K$ endowed with the restriction of the valuation $v$. Since the valuation will always be $v$, we only look for the subfields $E_{i}$. Let $\perp$ be the minimum of $I$. We find the fields $E_{i}$ by induction on $d(\perp, i)$ (note that $d(\perp, \top)=\max _{j \in I} d(\perp, j)$ ).

For $l \in\{0, \ldots, d(\perp, \top)\}$, let $D_{l}=\{i \in I \mid d(\perp, i)=l\}$.

If $d(\perp, i)=0$, then, by hypothesis and by the claim above, we already have the subvalued field $\left(E_{\perp}, v_{\perp}\right) \subseteq(K, v)$. Note that since $D_{0}=\{\perp\}$ the sequence of fields $\left(F_{i}\right)_{i \in D_{0}}$ is a good residue of $\left(E_{i}\right)_{i \in D_{0}}$ in $(K, v)$.

Assume we have found a system of fields $\left(E_{i}\right)_{i \in I, d(\perp, i) \leq l}$ such that $\operatorname{res}\left(E_{i}, v\right.$ 个 $\left.E_{i}\right)=F_{i}$ for $i \in I$ so that $d(\perp, i) \leq l$ and $\left(F_{i}\right)_{i \in D_{l}}$ is a good residue of $\left(E_{i}\right)_{i \in D_{l}}$ in $(K, v)$. We write $D_{l+1}=\left\{i_{k} \mid k<m\right\}$, then we apply Lemma 2.9 with $\left(F_{k}^{\prime}\right)_{k<m}=$ $\left(F_{i_{k}}\right)_{k<m}$, and obtain in this way a sequence $\left(E_{k}^{\prime}\right)_{k<m}$. We define the fields $E_{i}$ for $i \in D_{l+1}$ by $\left(E_{i_{k}}\right)_{k<m}=\left(E_{k}^{\prime}\right)_{k<m}$.

Finally, we can replace $\left(E_{\top}, v_{\top}\right)$ by one of it henselian closures, and each $\left(E_{i}, v_{i}\right)$ by its henselian closure inside $\left(E_{\top}, v_{\top}\right)$. The new residue system is isomorphic to the previously defined residue system, which shows that we can assume that all $\left(E_{i}, v_{i}\right)$ are henselian.

\section{Main results}

Our main result, Corollary 3.3, is a direct consequence of the next two theorems, whose proofs are given in Sections 4 and 5 respectively.

Theorem 3.1. Let $\mathscr{K}:=\left(K_{i}, f_{i j}\right)_{i \leq j \in I}$ be a projective system of fields (respectively formally real Pythagorean fields) such that $G\left(K_{i}\right)$ is finite for every $i \in I$. Let $\left(G_{i}, \lambda_{i j}\right)_{i \leq j \in I}=G(\mathscr{K})$ and let $G$ be the projective limit of this projective system of finite special groups. Then $G$ is isomorphic to the special group of some field (respectively formally real Pythagorean field).

Theorem 3.2. Let $G:=\left(G_{i}, \lambda_{i j}\right)_{i \leq j \in I}$ be an adequate projective system of finite reduced special groups (see Definition 2.4). Then there is a projective system $\mathscr{K}$ of formally real Pythagorean fields whose morphisms are inclusions, such that $\varphi \cong G(\mathscr{K})$.

Now consider a profinite reduced special group $G$. Say it is the projective limit of the system $\mathscr{G}=\left(G_{i}, f_{i j}\right)_{i \leq j \in I}$ of finite reduced special groups. Let $i^{\prime}$ be any 
element in $I$ and consider the system $\mathscr{G}^{\prime}$ equal to $\mathscr{G}$ restricted to indices in $I^{\prime}:=$ $\left\{i \in I \mid i \leq i^{\prime}\right\}$. The special group $G$ is the projective limit of the system $\mathscr{G}^{\prime}$, whose index set $I^{\prime}$ has a maximum element $T=i^{\prime}$. We can now use the strategy outlined after Definition 2.2 to express $G$ as an adequate projective system whose index set is $I^{\prime}$. Applying Theorem 3.2 then Theorem 3.1 now yields:

Corollary 3.3. Every profinite reduced special group is isomorphic to the special group of some formally real Pythagorean field.

\section{Proof of Theorem 3.1}

If $(I, \leq)$ is a downward directed poset and $i \in I$, then $i^{\leftarrow}$ denotes $\{j \in I \mid j \leq i\}$ and $i^{\rightarrow}$ denotes $\{j \in I \mid j \geq i\}$.

We first assume the following reductions:

(1) $I$ has a maximum $\top\left(I=\top^{\leftarrow}\right)$.

(2) All the $K_{i}, i \in I$, are subfields of the field $M:=K_{\top}$, and the morphisms $f_{i j}: K_{i} \rightarrow K_{j}$ are inclusions. In particular, the projective limit of the system $\mathscr{K}$ is isomorphic to the intersection of the fields $K_{i}, i \in I$.

These assumptions can safely be made because for the original projective system of fields $\mathscr{K}:=\left(K_{j}, f_{j k}\right)_{j \leq k \in I}$ and for each $i^{\prime} \in I$ fixed,

(i) the set $i^{\prime \leftarrow}$ is a coinitial subset of $I$, and

(ii) if $j \leq i^{\prime} \in I$, we can identify $K_{j}$ with the subfield $K_{j}^{\prime}:=f_{j i^{\prime}}\left[K_{j}\right]$ of $K_{i^{\prime}}$, and the morphisms $f_{j k}: K_{j} \rightarrow K_{k}$ are naturally identified with inclusions $\iota_{j k}: K_{j}^{\prime} \hookrightarrow K_{k}^{\prime}$.

The reductions above give us

$$
\begin{aligned}
\lim _{(}\left(K_{j}, f_{j k}\right)_{j \leq k \in I} \cong \lim _{\longleftarrow}\left(K_{j}, f_{j k}\right)_{j \leq k \in i^{\prime}} \leftarrow & \cong \lim _{\longleftarrow}\left(K_{j}^{\prime}, \iota_{j k}\right)_{j \leq k \in i^{\prime}} \\
& \cong \bigcap_{j \in i^{\prime}} f_{j i^{\prime}}\left[K_{j}\right] \subseteq K_{i^{\prime}}
\end{aligned}
$$

and

$$
\begin{aligned}
G:=\lim _{\longleftarrow}\left(G\left(K_{j}\right), G\left(f_{j k}\right)\right)_{j \leq k \in I} & \cong \lim _{\longleftarrow}\left(G\left(K_{j}\right), G\left(f_{j k}\right)\right)_{j \leq k \in i^{\prime}} \leftarrow \\
& \cong \lim _{\longleftarrow}\left(G\left(K_{j}^{\prime}\right), G\left(\iota_{j k}\right)\right)_{j \leq k \in i^{\prime} \leftarrow} .
\end{aligned}
$$

Now consider the language $L=L_{R} \cup\left\{R_{i}\right\}_{i \in I}$, where $L_{R}$ is the language of rings and the $R_{i}$ are unary relation symbols. We turn $M$ into an $L$-structure by interpreting each $R_{i}$ in $M$ by the subfield $K_{i}$.

Let $N$ be an $|I|^{+}$-saturated elementary extension of $M$ in the language $L$. (See [Chang and Keisler 1990, Chapter 5 and Lemma 5.1.2] or [Hodges 1993, p. 480 and Corollary 10.2.2] for the definition of saturated models and the existence result we just used. Note that this notion of saturation is not linked to the existing one 
for subgroups of special groups.) Each $F_{i}:=R_{i}^{N}$ is a subfield of $N$, and the

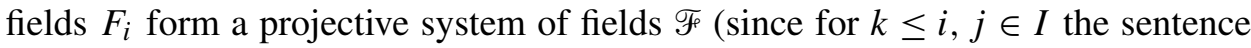
" $R_{k} \subseteq R_{i} \cap R_{j}$ " is in the theory of $M$ ). Moreover, for every $i \in I, K_{i} \hookrightarrow F_{i}$ is an $L_{R}$-elementary embedding and therefore induces an isomorphism of special groups

$$
G\left(K_{i}\right) \stackrel{\cong}{\longrightarrow} G\left(F_{i}\right)
$$

(since the special groups $G\left(K_{i}\right)$, being finite, are described in the theory of $M$ ). More generally $G(\mathscr{K}) \cong G(\mathscr{F})$, so $G \cong \lim G(\mathscr{K}) \cong \lim G(\mathscr{F})$.

Let $F:=\bigcap_{i \in I} F_{i}$ and define

$$
\begin{aligned}
\xi: G(F) & \left.\rightarrow \underset{\lim G(\mathscr{F})}{\longleftarrow} \dot{F}^{2}\right)_{i \in I} .
\end{aligned}
$$

We show that $\xi$ is an isomorphism of special groups, which yields $G \cong G(F)$ as needed (in particular, if the fields $K_{i}, i \in I$, are formally real Pythagorean, then $F=\bigcap_{i \in I} F_{i}$ is formally real Pythagorean, since $G(F)$ is a reduced special group).

Step 1. It is clear that $\xi$ is well-defined and is a morphism of groups.

Step 2. $\xi$ is a morphism of special groups. Indeed, it is clear that $\xi$ sends -1 to

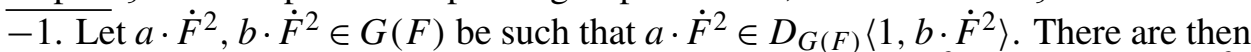
$c, d \in F$ such that, for all $i \in I, a=c^{2}+b d^{2}$ in $F_{i}$. Then $a \cdot \dot{F}_{i}{ }^{2} \in D_{G\left(F_{i}\right)}\left\langle 1, b \cdot \dot{F}_{i}{ }^{2}\right\rangle$ for every $i \in I$, and therefore $\xi\left(a \cdot \dot{F}^{2}\right) \in D_{G^{\prime}}\left\langle 1, \xi\left(b \cdot \dot{F}^{2}\right)\right\rangle$.

Step 3. $\xi$ is surjective: Let $a=\left(a_{i} \cdot \dot{F}_{i}^{2}\right)_{i \in I} \in \lim G\left(F_{i}\right)$. So for all $i \leq j \in I$, $\overline{a_{i} \cdot \dot{F}_{j}^{2}}=a_{j} \cdot \dot{F}_{j}^{2}$. We want $x \in N$ satisfying the set of formulas

$$
\Delta:=\left\{x \in F_{i}\right\}_{i \in I} \cup\left\{x=a_{i} \bmod \dot{F}_{i}^{2}\right\}_{i \in I} .
$$

Every finite part of $\Delta$ is satisfied in $N$ since $a=\left(a_{i} \cdot \dot{F}_{i}^{2}\right)_{i \in I} \in \lim G\left(F_{i}\right)$ (it suffices to take $x=a_{k}$, where $k$ is less than every one of the indices $i \in I$ occurring in this finite part). By $|I|^{+}$-saturation, $\Delta$ has a solution $x$ in $N$. Then $\xi(x)=\left(a_{i} \cdot \dot{F}_{i}^{2}\right)$.

The rest of the proof relies on the following lemma.

Lemma 4.1. Let $n \in \mathbb{N}$ and let $P\left(X_{1}, \ldots, X_{n}\right) \in F\left[X_{1}, \ldots, X_{n}\right]$. Assume the equation $P\left(X_{1}, \ldots, X_{n}\right)=0$ has a solution in every $F_{i}, i \in I$. Then the same equation has a solution in $F$.

Proof. We are looking for $\bar{x} \in N$ such that the set of formulas

$$
\Sigma:=\{P(\bar{x})=0\} \cup\left\{\bar{x} \in F_{i}\right\}_{i \in I}
$$

is satisfied in $N$. Since the $F_{i}$, together with the inclusions between them, form a projective system, every finite part of $\Sigma$ has a solution, and by the $|I|^{+}$-saturation of $N, \Sigma$ has a solution in $N$. 
We go back to proving that $\xi$ is an isomorphism:

Step 4. $\xi$ is injective: Let $a=a \cdot \dot{F}^{2} \in G(F)$ be such that $\xi(a)=1$, i.e., $a \in \dot{F}_{i}^{2}$ for every $i \in I$, i.e., the polynomial $X^{2}-a$ has a root in each $F_{i}, i \in I$. By Lemma 4.1, $X^{2}-a$ has a root in $F$, hence $a \in \dot{F}^{2}$.

Step 5. $\xi$ is a monomorphism of special groups: Let $a, b \in F$ be such that, for $\overline{\text { every } i} \in I, a \cdot \dot{F}_{i}^{2} \in D\left\langle 1, b \cdot \dot{F}_{i}^{2}\right\rangle$. Let $P(X, Y)=a-\left(X^{2}+b Y^{2}\right) \in F[X, Y]$. By hypothesis, $P(X, Y)=0$ has a solution in each $F_{i}$, hence a solution in $F$ by Lemma 4.1, which means $a \in D_{G(F)}\langle 1, b\rangle$.

\section{Proof of Theorem 3.2}

Reducing to a finite projective system. Since $\mathscr{G}$ is adequate, the set $(I, \leq)$ has a maximum element $T$.

In this subsection we show that it is enough to prove Theorem 3.2 when $\varphi:=$ $\left(G_{i}, f_{i j}\right)_{i \leq j \in I}$ is a finite projective system of special groups such that $I$ has a maximum (which we will also denote by $T$ ).

Let $L$ be the language $\{0,1,-,+, \cdot\} \cup\left\{F_{i} \mid i \in I\right\} \cup\left\{Q_{i}^{g} \mid i \in I, g \in G_{i}\right\}$, where 0,1 are constant symbols, - is a unary function symbol,,$+ \cdot$ are binary function symbols and $F_{i}, Q_{i}^{g}$ are unary predicate symbols, for each $i \in I$ and $g \in G_{i}$. Denote by $\lambda_{i}$ the inverse of the bijection $g \in G_{i} \mapsto Q_{i}^{g}, i \in I$. The projective system of fields we are looking for is a model of the theory $\Omega$ consisting of (first-order) $L$-sentences that are informally described in the four items below:

(1) the interpretation of the unary predicate $F_{\top}$ is the universe of the $L$-structure (i.e., $\left.\forall x\left(F_{\top}(x)\right)\right)$ and " $\left(F_{\top}, 0,1,+, \cdot\right)$ is a field";

(2) for every $i \leq j \in I$ :

" $F_{i} \subseteq F_{j}$ " and " $\left(F_{i}, 0,1,+, \cdot\right)$ is a subfield of the field $\left(F_{\mathrm{T}}, 0,1,+, \cdot\right)$ " (technically speaking, + and $\cdot$ are functional symbols globally defined whose restrictions to $F_{i}$ give internal operations on $F_{i}$ );

(3) for every $i \in I$ :

" $\lambda_{i}$ is an isomorphism of special groups $G\left(F_{i}\right) \rightarrow G_{i}$ ";

(4) for every $i \leq j \in I$ :

"the morphism of special groups induced by the inclusion $F_{i} \subseteq F_{j}$ is naturally identified with $f_{i j}$, via the isomorphisms $\lambda_{i}, \lambda_{j}$ ".

It is clear how to describe the expressions in items (1) and (2) by first-order $L$ sentences. For the reader's convenience, we add a more explicit description of the $L$-sentences involved in the two remaining items: the hypothesis that the special groups $G_{i}$ are all finite ensures that the prescription in item (3) can be encoded by a set of first-order $L$-sentences. 
Item (3): for each $i \in I$ :

- for each $g \in G_{i}$, " $Q_{i}^{g} \subseteq \dot{F}_{i}$ " and " $Q_{i}^{g}=a \dot{F}_{i}^{2}$, for some $a \in \dot{F}_{i}$ ";

- for each $g, g^{\prime} \in G_{i}$ such that $g \neq g^{\prime}$, " $Q_{i}^{g} \cap Q_{i}^{g^{\prime}}=\varnothing ”$;

- " $\dot{F}_{i}=\bigcup\left\{Q_{i}^{g} \mid g \in G_{i}\right\}$ " (as $G_{i}$ is a finite special group, this can be described by a first-order $L$-sentence);

- " $1 \in Q_{i}^{1}$ and $-1 \in Q_{i}^{-1}$ ";

- for each $g, g^{\prime} \in G_{i}$, "for each $a, a^{\prime}$, if $a \in Q_{i}^{g}$ and $a^{\prime} \in Q_{i}^{g^{\prime}}$ then $a \cdot a^{\prime} \in Q_{i}^{g g^{\prime}}$ ";

- for each $g, g^{\prime} \in G_{i}$ such that $g^{\prime} \in D_{G_{i}}(1, g)$, "for each $a, a^{\prime}$, if $a \in Q_{i}^{g}$ and $a^{\prime} \in Q_{i}^{g^{\prime}}$ then there are $x, y \in F_{i}$ such that $a^{\prime}=x^{2}+a y^{2}$ ";

- for each $g, g^{\prime} \in G_{i}$ such that $g^{\prime} \notin D_{G_{i}}(1, g)$, "for each $a, a^{\prime}$, if $a \in Q_{i}^{g}$ and $a^{\prime} \in Q_{i}^{g^{\prime}}$ then for all $x, y \in F_{i}, a^{\prime} \neq x^{2}+a y^{2}$ ".

Item (4): for each $i \leq j \in I$ :

By the axioms above: since $F_{i} \subseteq F_{j}$ and we have the partitions

$$
\dot{F}_{i} / \dot{F}_{i}^{2}=\left\{Q_{i}^{g} \mid g \in G_{i}\right\} \quad \text { and } \quad \dot{F}_{j} / \dot{F}_{j}^{2}=\left\{Q_{j}^{g^{\prime}} \mid g^{\prime} \in G_{j}\right\},
$$

then for each $g \in G_{i}$ there is a unique $g^{\prime} \in G_{j}$ such that $Q_{i}^{g} \subseteq Q_{j}^{g^{\prime}}$. In this way we obtain a function $q_{i j}: \dot{F}_{i} / \dot{F}_{i}^{2} \rightarrow \dot{F}_{j} / \dot{F}_{j}^{2}$. Clearly $q_{i j}\left(a . \dot{F}_{i}^{2}\right)=a \cdot \dot{F}_{j}^{2}$, for every $a \in \dot{F}_{i}$, i.e., $q_{i j}$ is the special group morphism induced by the inclusion $F_{i} \subseteq F_{j}$. We add a new list of axioms expressing that $\lambda_{j} \circ q_{i j}=f_{i j} \circ \lambda_{i}$. A direct examination of the equivalent condition $q_{i j}=\lambda_{j}^{-1} \circ f_{i j} \circ \lambda_{i}$ shows that these axioms must be

$$
\text { for each } g \in G_{i} \text {, " } Q_{i}^{g} \subseteq Q_{j}^{f_{i j}(g),} \text {. }
$$

Using now the compactness theorem (see [Chang and Keisler 1990, Theorem 1.3.22] or [Hodges 1993, Theorem 6.1.1]), to find a model of this theory we only need to find a model of every finite part $\Omega_{0} \subseteq \Omega$. Let $J$ be the set of elements of $I$ occurring in this finite part $\Omega_{0}$, together with $\top$. Since $I$ is downward directed, we can assume that $J$ is also downward directed (taking a larger set $J$ if necessary), that is $J$ has a first element $\perp$. In particular $J$ determines a finite projective system of special groups whose index set that has a maximum and a minimum.

Description of the proof by induction. We therefore assume from now on that the index poset $(I, \leq)$ is finite and that it has a minimum $\perp$ and a maximum $\top$. We find a finite projective system $\mathscr{K}$ of Pythagorean fields of characteristic 0 such that $\mathscr{G} \cong G(\mathscr{K})$ by induction on the construction of $G_{\perp}$ by products and extensions. For the purpose of the proof, we allow the (nonreduced) special group $\{1\}$ to appear in $\varphi$.

Recall that since $\mathscr{G}$ is an adequate projective system, the morphisms $f_{i j}, i \leq j \in I$, are quotients by saturated subgroups (see the paragraph after Definition 2.2). 
If $G_{\perp} \cong\{1\}$, then all special groups in the system are trivial and all morphisms are isomorphisms. We can obviously realize such a system by taking $F_{i}=A, i \in I$, where $A$ is any fixed algebraically closed field of characteristic 0 .

If $G_{\perp} \cong \mathbb{Z}_{2}$, then all special groups in the system are isomorphic to $\mathbb{Z}_{2}$ or to $\{1\}$ and all morphisms are isomorphisms or naturally identified with $\mathbb{Z}_{2} \rightarrow\{1\}$. We can obviously realize such a system by simply selecting a real closed field $R$ and an algebraically closed field $A$ such that $R \subseteq A$.

If $G_{\perp} \cong G_{\perp}^{\prime} \times G_{\perp}^{\prime \prime}$, since all morphisms and special groups in the systems are quotients of $G_{\perp}$ by (larger and larger) saturated subgroups, and using Fact 2.5, the whole projective system $\left(G_{i}, f_{i j}\right)_{i \leq j \in I}$ splits according to the product $G_{\perp} \cong$ $G_{\perp}^{\prime} \times G_{\perp}^{\prime \prime}$ into two adequate projective systems of finite special groups:

$$
\left(G_{i}^{\prime}, f_{i j}^{\prime}\right)_{i \leq j \in I} \text { and }\left(G_{i}^{\prime \prime}, f_{i j}^{\prime \prime}\right)_{i \leq j \in I} .
$$

(Note that, for each $i \in I$, if $G_{i}$ is reduced, then either both $G_{i}^{\prime}$ and $G_{i}^{\prime \prime}$ are reduced or one of them is the trivial special group $\{1\}$ and the other is reduced.) By induction the systems in (5-1) are realized by two projective systems of Pythagorean fields of characteristic 0: $\mathscr{F}^{\prime}=\left(F_{i}^{\prime}\right)_{i \in I}$ and $\mathscr{F}^{\prime \prime}=\left(F_{i}^{\prime \prime}\right)_{i \in I}$ (where the morphisms are inclusions), so we just need to "glue" them together. For this we use results from [Kula 1979], which describe how to realize a finite product of finite special groups when each one is already realized. This is achieved in the next subsection.

If $G_{\perp} \cong G^{\prime}[H]$, as above, the morphisms of special groups in the projective system are quotients of $G_{\perp}$ by (larger and larger) saturated subgroups $\Delta_{i}$. This case is dealt with starting on page 279, using results from [Becher 2002].

Gluing, the product case. The next several pages are taken by the proof of the following result.

Theorem 5.1. Let $(I, \leq)$ be a finite downward directed index set with first element $\perp$ and last element $\mathrm{T}$. Let $\mathscr{F}^{\prime}=\left(F_{i}^{\prime}\right)_{i \in I}, \mathscr{F}^{\prime \prime}=\left(F_{i}^{\prime \prime}\right)_{i \in I}$ be finite projective systems of fields of characteristic 0 , where the morphisms are inclusions and such that for every $i \in I G\left(F_{i}^{\prime}\right)$ and $G\left(F_{i}^{\prime \prime}\right)$ are finite special groups. Then there is a finite projective system $\mathscr{F}=\left(F_{i}\right)_{i \in I}$ of fields of characteristic 0 (where the morphisms are inclusions) such that

$$
G\left(\mathscr{F}^{\prime}\right) \cong G\left(\mathscr{F}^{\prime}\right) \times G\left(\mathscr{F}^{\prime \prime}\right) .
$$

Remark 5.2. In this theorem, for each $i \in I$ we have:

(a) $F_{i}$ is Pythagorean if and only if $F_{i}^{\prime}$ and $F_{i}^{\prime \prime}$ are Pythagorean.

(b) If $F_{i}$ is Pythagorean, then $F_{i}$ is formally real if and only if $F_{i}^{\prime}$ or $F_{i}^{\prime \prime}$ is formally real.

We begin with a reformulation of some results from [Kula 1979]. 
Definition 5.3. Let $F$ be a field equipped with $n$ mutually independent valuations of rank one $v_{1}, \ldots, v_{n}$, and let $f_{i}$ be an embedding of $F$ into $F^{v_{i}}$, a completion of $F$ with respect to $v_{i}$. We say that $\left(F, f_{1}, \ldots, f_{n}\right)$ fulfills the global squares property if, for every $a \in F$,

$$
a \in \dot{F}^{2} \Longleftrightarrow \forall i \in\{1, \ldots, n\} f_{i}(a) \in\left(\dot{F}^{v_{i}}\right)^{2} .
$$

(Note that the left to right implication always holds.)

Theorem 5.4 [Kula 1979, Corollary 2.5]. With notation as in Definition 5.3, assume that $\left(F, f_{1}, \ldots, f_{n}\right)$ fulfills the global squares property. Then the map

$$
\begin{aligned}
\xi_{F}: G(F) & \rightarrow \prod_{i=1}^{n} G\left(F^{v_{i}}\right) \\
a \dot{F}^{2} & \mapsto\left(f_{i}(a) \cdot\left(\dot{F}^{v_{i}}\right)^{2}\right)_{i=1, \ldots, n}
\end{aligned}
$$

is an isomorphism of special groups.

Theorem 5.5 [Kula 1979, Theorem 2.6]. Let $\left(L_{i}, v_{i 1}, \ldots, v_{i n}\right)_{i \in I}$ be a finite projective system of fields equipped with $n$ mutually independent valuations of rank one, and such that I has a maximum element $T$. Then for every $i \in I$ there is an algebraic extension $\mathscr{E}\left(L_{i}\right)$ of $L_{i}$ and a morphism of special groups

$$
\eta_{i}: G\left(\mathscr{E}\left(L_{i}\right)\right) \rightarrow \prod_{k=1}^{n} G\left(\left(L_{i}\right)^{v_{i k}}\right)
$$

such that

(1) $\mathscr{E}\left(L_{i}\right) \subseteq \mathscr{E}\left(L_{j}\right)$ for every $j \in I, j \geq i$;

(2) $G\left(\mathscr{E}\left(L_{i}\right)\right) \underset{\overline{\eta_{i}}}{\cong} \prod_{k=1}^{n} G\left(\left(L_{i}\right)^{v_{i k}}\right)$; and

(3) the morphism of special groups $\prod_{k=1}^{n} G\left(\left(L_{i}\right)^{v_{i k}}\right) \rightarrow \prod_{k=1}^{n} G\left(\left(L_{j}\right)^{v_{j k}}\right)$, given by the product of the morphisms of special groups induced by $\left(L_{i}\right)^{v_{i k}} \subseteq\left(L_{j}\right)^{v_{j k}}$ is naturally identified, via the isomorphisms $\eta_{i}$ and $\eta_{j}$, with the morphism of special groups $G\left(\mathscr{E}\left(L_{i}\right)\right) \rightarrow G\left(\mathscr{E}\left(L_{j}\right)\right)$ induced by $\mathscr{E}\left(L_{i}\right) \subseteq \mathscr{E}\left(L_{j}\right)$.

Proof. Since a valuation $v_{i k}$ is the restriction on $L_{i}$ of the valuation $v_{\top k}$, we drop the first index and simply denote it by $v_{k}$. For $k \in\{1, \ldots, n\}$ we fix a completion $L_{\top}^{k}$ of $L_{\top}$ with respect to $v_{k}$ and define, for $i \in I, L_{i}^{k}$ to be the completion of $L_{i}$ in $L_{\top}^{k}$ with respect to $v_{k}$. The systems $\left(L_{i}^{k}\right)_{i \in I}$, for $k \in\{1, \ldots, n\}$, are all projective systems of fields, where the morphisms are the inclusions (since $L_{i}^{k}$ is simply the set of limits in $L_{\top}^{k}$ of $v_{k}$-Cauchy sequences of elements of $L_{i}$ ).

Let $K_{+}$be an algebraic closure of $L_{\top}$. We define the set

$\mathscr{L}:=\left\{\right.$ projective systems of fields $\left(E_{i}, \iota_{i 1}, \ldots, \iota_{i n}\right)_{i \in I}$ such that $L_{i} \subseteq E_{i} \subseteq K_{+}$with $E_{i} \mid L_{i}$ algebraic, equipped with the $L_{i}$-embeddings of fields $\iota_{i k}: E_{i} \rightarrow L_{i}^{k}$ for $\left.k=1, \ldots, n\right\}$. 
(Note that the condition that $\left(E_{i}, \iota_{i 1}, \ldots, \iota_{i n}\right)_{i \in I}$ is a projective system implies $\iota_{i k} \subseteq \iota_{j k}$ for $i \leq j \in I$ and $k \in\{1, \ldots, n\}$, which is possible since $L_{i}^{k} \subseteq L_{j}^{k}$.) We equip $\mathscr{L}$ with the partial ordering

$$
\begin{gathered}
\left(E_{i}, \iota_{i 1}, \ldots, \iota_{i n}\right)_{i \in I} \leq\left(F_{i}, \kappa_{i 1}, \ldots, \kappa_{i n}\right)_{i \in I} \\
\text { if and only if }
\end{gathered}
$$

for every $i \in I$ and $k \in\{1, \ldots, n\}, E_{i} \subseteq F_{i}$ and $\iota_{i k} \subseteq \kappa_{i k}$.

By Zorn's lemma, $\mathscr{L}$ has a maximal element $\left(M_{i}, f_{i 1}, \ldots, f_{i n}\right)_{i \in I}$. We show that, for $j \in I,\left(M_{j}, f_{j 1}, \ldots, f_{j n}\right)$ fulfills the global squares property. Let $j \in I$ and let $a \in M_{j} \backslash\{0\}$ be such that $f_{j k}(a) \in\left(L_{j}^{k}\right)^{\times 2}$, for $k=1, \ldots, n$. Assume $\sqrt{a} \notin M_{j}$. Fix a square root $\sqrt{a}$ of $a$ and $\alpha_{k} \in L_{j}^{k}$ such that $\alpha_{k}^{2}=f_{j k}(a)$. Then each morphism $f_{j k}$ can be (properly) extended to $M_{j}^{\prime}:=M_{j}(\sqrt{a})$ by sending $\sqrt{a}$ to $\alpha_{k}$. Moreover, with $A_{j}:=\{r \in I \mid r \geq j\}$, and since for $r \in A_{j}$ we have $L_{j}^{k} \subseteq L_{r}^{k}$, the same reasoning tells us that, for each $r \in A_{j}$ and $k \in\{1, \ldots, n\}$, each morphism $f_{r k}$ can be extended to $M_{r}^{\prime}:=M_{r}(\sqrt{a})$ by sending $\sqrt{a}$ to $\alpha_{k}$ (since $\alpha_{k} \in L_{r}^{k}$ ). If $r \in I \backslash A_{j}$, we take $M_{r}^{\prime}:=M_{r}$. We obtain in this way $\left(M_{i}^{\prime}, f_{i}^{\prime}, \ldots, f_{n}^{\prime}\right)_{i \in I}$, a projective system of fields equipped with $n$ morphisms of fields that is (strictly) larger than $\left(M_{i}, f_{i}, \ldots, f_{n}\right)_{i \in I}$, a contradiction. It follows that $\sqrt{a} \in M_{j}$ and thus that $\left(M_{j}, f_{j 1}, \ldots, f_{j n}\right)$, for $j \in I$, fulfills the global squares property. If we take $\mathscr{E}\left(L_{i}\right)=M_{i}$ for $i \in I$, the first conclusion of the theorem then holds, and the second follows by Theorem 5.4, with

$$
\begin{aligned}
\eta_{i}: G\left(M_{i}\right) & \cong \prod_{k=1}^{n} G\left(\left(L_{i}\right)^{k}\right) \\
a \cdot \dot{M}_{i}^{2} & \mapsto\left(f_{i}(a) \cdot\left(L_{i}^{k}\right)^{\times 2}\right)_{i=1, \ldots, n},
\end{aligned}
$$

for $i \in I$. The third conclusion is proved in the next lemma.

Lemma 5.6. Let the notation be as in Theorem 5.5 and its proof.

Let $\left(L, v_{1}, \ldots, v_{n}\right) \supseteq\left(K, v_{1}\left\lceil K, \ldots, v_{n}\lceil K)\right.\right.$ be two fields equipped with $n$ mutually independent valuations of rank one. For $m=1, \ldots, n$ let

- $L^{m}$ be a completion of $L$ with respect to $v_{m}$ and $K^{m}$ be a completion of $K$ with respect to $v_{m}\left\lceil K\right.$ such that $K^{m} \subseteq L^{m}$,

- $f_{m}$ be an embedding of $K$ into $K^{m}$ and $g_{m}$ be an embedding of $L$ into $L^{m}$ extending $f_{m}$.

Assume $\left(K, f_{1}, \ldots, f_{n}\right)$ and $\left(L, g_{1}, \ldots, g_{n}\right)$ satisfy the global squares property.

Let $\lambda: \prod_{m=1}^{n} G\left(K^{m}\right) \rightarrow \prod_{m=1}^{n} G\left(L^{m}\right)$ be the product of the morphisms of special groups induced by the inclusions $K^{m} \subseteq L^{m}$ for $m=1, \ldots, n$, and let $\mu: G(K) \rightarrow G(L)$ be the morphism of special groups induced by $K \subseteq L$.

Then $\lambda$ and $\mu$ are naturally identified via the isomorphisms $\xi_{K}$ and $\xi_{L}$ given by Theorem 5.4. 
Proof. By Theorem 5.4 the isomorphism $G(K) \cong G\left(K^{1}\right) \times \cdots \times G\left(K^{n}\right)$ is

$$
\begin{aligned}
\xi_{K}: G(K) & \rightarrow G\left(K^{1}\right) \times \cdots \times G\left(K^{n}\right) \\
x \cdot \dot{K}^{2} & \mapsto\left(f_{1}(x) \cdot\left(\dot{K}^{1}\right)^{2}, \ldots, f_{n}(x) \cdot\left(\dot{K}^{n}\right)^{2}\right) .
\end{aligned}
$$

Similarly, the isomorphism between $G(L)$ and $G\left(L^{1}\right) \times \cdots \times G\left(L^{n}\right)$ is

$$
\begin{aligned}
\xi_{L}: G(L) & \rightarrow G\left(L^{1}\right) \times \cdots \times G\left(L^{n}\right) \\
x \cdot \dot{L}^{2} & \mapsto\left(g_{1}(x) \cdot\left(\dot{L^{1}}\right)^{2}, \ldots, g_{n}(x) \cdot\left(\dot{L^{n}}\right)^{2}\right) .
\end{aligned}
$$

Thus $\lambda=\xi_{L} \circ \mu \circ \xi_{K}^{-1}$ since $g_{m} \uparrow K=f_{m}$ for $m=1, \ldots, n$.

We now turn our attention to the two finite projective systems of fields $\mathscr{F}^{\prime}=$ $\left(F_{i}^{\prime}\right)_{i \in I}$ and $\mathscr{F}^{\prime \prime}=\left(F_{i}^{\prime \prime}\right)_{i \in I}$ of characteristic zero introduced in the statement of Theorem 5.1. We first show that we can assume that the fields in $\mathscr{F}^{\prime}$ and $\mathscr{F}^{\prime \prime}$ are at most countable and of finite transcendence degree over $\mathbb{Q}$. This is achieved by the following proposition.

Proposition 5.7 [Kula 1979, Proposition 3.1]. Let $\mathscr{L}:=\left(L_{i}\right)_{i \in I}$ be a finite projective system of fields of characteristic 0 such that $G\left(L_{i}\right)$ is a finite special group for all $i \in I$. There is a map $\mathscr{F}$, defined on $\left\{L_{i}\right\}_{i \in I}$, satisfying the following properties whenever $i \leq j \in I$ :

(1) $\mathscr{F}\left(L_{i}\right)$ is a countable subfield of $L_{i}$ with finite transcendence degree over $\mathbb{Q}$.

(2) If $\varphi_{i}: \mathscr{F}\left(L_{i}\right) \hookrightarrow L_{i}$ is the inclusion map, then $G\left(\varphi_{i}\right): G\left(\mathscr{F}\left(L_{i}\right)\right) \rightarrow G\left(L_{i}\right)$ is an isomorphism of special groups.

(3) $\mathscr{F}\left(L_{i}\right) \subseteq \mathscr{F}\left(L_{j}\right)$.

(4) If $\lambda_{i j}: G\left(L_{i}\right) \rightarrow G\left(L_{j}\right)$ is the morphism of special groups induced by $L_{i} \subseteq$ $L_{j}$, then the morphism of special groups $G\left(\mathscr{F}\left(L_{i}\right)\right) \rightarrow G\left(\mathscr{F}\left(L_{j}\right)\right)$ induced by $\mathscr{F}\left(L_{i}\right) \subseteq \mathscr{F}\left(L_{j}\right)$ is naturally identified with $\lambda_{i j}$, via the isomorphisms $G\left(\varphi_{i}\right)$ and $G\left(\varphi_{j}\right)$.

Proof. The proof is a trivial extension of Kula's. If $L$ is a field with a finite number of square classes, a representative system of $G(L)$ is a finite subset $R(L)=A \cup B$ of $L$ such that

- $A \subseteq \dot{L}$ and $\dot{L} / \dot{L}^{2}=A / \dot{L}^{2}$;

- For every $a_{1}, a_{2} \in A$ with $a_{1} \in D_{L}\left\langle 1, a_{2}\right\rangle$, there are $b_{1}, b_{2} \in B$ such that $a_{1}=b_{1}^{2}+a_{2} b_{2}^{2}$.

Claim: For every $i \in I$ there is a representative system $R\left(L_{i}\right)$ of $L_{i}$ such that $R\left(L_{i}\right) \subseteq R\left(L_{j}\right)$ whenever $i \leq j$.

Proof of the claim: Direct by induction on $d(\perp, i)$ (just take a system of representatives of $L_{i}$ and add to it all the $R\left(L_{j}\right)$ for $\left.\perp \leq j<i\right)$. 
Then, just as in [Kula 1979], take for $\mathscr{F}\left(L_{i}\right)$ the algebraic closure of $\mathbb{Q}\left(R\left(L_{i}\right)\right)$ in $L_{i}$.

The following two propositions show that we can assume that $\operatorname{atd}\left(F_{i}^{\prime}\right)=\operatorname{atd}\left(F_{i}^{\prime \prime}\right)$ for every $i \in I$, where atd denotes the absolute transcendence degree, i.e., the transcendence degree over $\mathbb{Q}$.

Proposition 5.8 ([Kula 1979], Lemma 3.2). Let $\left(L_{i}\right)_{i \in I}$ be a finite projective system of countable fields of finite absolute transcendence degree. There is a map $\mathcal{T}$ defined on $\left\{L_{i}\right\}_{i \in I}$ satisfying the following properties whenever $i \leq j \in I$ :

(1) $\mathscr{T}\left(L_{i}\right)$ is a countable field extension of $L_{i}$.

(2) $\operatorname{atd}\left(\mathscr{T}\left(L_{i}\right)\right)=\operatorname{atd}\left(L_{i}\right)+1$.

(3) If $\tau_{i}: L_{i} \hookrightarrow \mathscr{T}\left(L_{i}\right)$ is the inclusion map, then $G\left(\tau_{i}\right): G\left(L_{i}\right) \rightarrow G\left(\mathscr{T}\left(L_{i}\right)\right)$ is an isomorphism of special groups.

(4) $\mathscr{T}\left(L_{i}\right) \subseteq \mathscr{T}\left(L_{j}\right)$.

(5) If $\lambda_{i j}: G\left(L_{i}\right) \rightarrow G\left(L_{j}\right)$ is the morphism of special groups induced by $L_{i} \subseteq$ $L_{j}$, then the morphism of special groups $G\left(\mathscr{T}\left(L_{i}\right)\right) \rightarrow G\left(\mathscr{T}\left(L_{j}\right)\right)$ induced by $\mathscr{T}\left(L_{i}\right) \subseteq \mathscr{T}\left(L_{j}\right)$ is naturally identified with $\lambda_{i j}$ (via $G\left(\tau_{i}\right)$ and $G\left(\tau_{j}\right)$ ).

Proof. For $i \in I$ let $K_{i}:=L_{i}(x)\left(\sqrt[2^{n}]{x}\right)_{n \in \mathbb{N}}(x$ is an indeterminate), and consider on $K_{i}$ the unique extension $v_{i}$ of the valuation on $L_{i}(x)$ determined by the irreducible polynomial $x$. The $K_{i}$, together with their inclusions, form a projective system, and the sets $\Phi_{i}:=\left\{v_{i}\right\}$ satisfy the hypothesis of Theorem 5.5. We now apply the map $\mathscr{E}$ defined in Theorem 5.5 to the projective system of the $K_{i}$ and get the projective system of the $\mathscr{T}\left(L_{i}\right)$. Since $L_{i}$ is countable, $K_{i}$ and $\mathscr{T}\left(L_{i}\right)=\mathscr{E}\left(K_{i}\right)$ are countable. Kula's proof of [Kula 1979, lemma 3.2] shows that the second and third claims of the proposition hold, and the last two hold by Theorem 5.5.

Proposition 5.9. There exist finite projective systems $\mathscr{K}^{\prime}=\left(K_{i}^{\prime}\right)_{i \in I}$ and $\mathscr{K}^{\prime \prime}=\left(K_{i}^{\prime \prime}\right)_{i \in I}$ of fields of characteristic 0 such that

(1) $G\left(\mathscr{K}^{\prime}\right) \cong G\left(\mathscr{F}^{\prime}\right)$ and $G\left(\mathscr{K}^{\prime \prime}\right) \cong G\left(\mathscr{F}^{\prime \prime}\right)$, and

(2) for every $i \in I, \operatorname{atd}\left(K_{i}^{\prime}\right)=\operatorname{atd}\left(K_{i}^{\prime \prime}\right)<\infty$.

Proof. We assume there is some $i \in I$ such that $\operatorname{atd}\left(F_{i}^{\prime}\right) \neq \operatorname{atd}\left(F_{i}^{\prime \prime}\right)$ and we proceed by induction on $d(\perp, i)$, the maximal length of a chain from $\perp$ to $i$.

- $d(\perp, i)=0$, i.e., $i=\perp$. Let $t:=\max \left\{\operatorname{atd}\left(F_{\perp}^{\prime}\right), \operatorname{atd}\left(F_{\perp}^{\prime \prime}\right)\right\}$. We then apply Proposition 5.8 as many times as necessary to the system $\mathscr{F}^{\prime}$ or $\mathscr{F}^{\prime \prime}$ (the one that does no realize the maximum), and we obtain two new systems $\mathscr{F}_{(0)}^{\prime}$ and $\mathscr{F}_{(0)}^{\prime \prime}$ indexed by $I$, whose fields of index $\perp$ have same (finite) absolute transcendence degree $t$. 
- $d(\perp, i)=n>0$. We now proceed by induction on the number of $i$ 's with $d(\perp, i)=n$ and $\operatorname{atd}\left(F_{i}^{\prime}\right) \neq \operatorname{atd}\left(F_{i}^{\prime \prime}\right)$. We fix one of them: $i_{1}$. By induction we can assume that the projective systems $\mathscr{F}^{\prime}$ and $\mathscr{F}^{\prime \prime} \operatorname{satisfy} \operatorname{atd}\left(F_{j}^{\prime}\right)=\operatorname{atd}\left(F_{j}^{\prime \prime}\right)$ for every $j \in I, d(\perp, j)<n$. We consider the systems $\mathscr{F}^{\prime}\left\lceil i_{1}\right.$ and $\mathscr{F}^{\prime \prime}\left\lceil i_{1}\right.$. By applying Proposition 5.8, we get two new systems $\mathscr{T}^{\prime}$ and $\mathscr{T}^{\prime \prime}$, indexed by $i_{1} \rightarrow$ whose fields indexed by $i_{1}$ have same absolute transcendence degree. We replace, in $\mathscr{F}^{\prime}$, respectively $\mathscr{F}^{\prime \prime}$, the subsystem $\mathscr{F}^{\prime}\left\lceil i_{1}\right.$ by $\mathscr{T}^{\prime}$, respectively $\mathscr{F}^{\prime \prime} \uparrow$ $i_{1}$ by $\mathscr{T}^{\prime \prime}$ and we write $\mathscr{F}_{(1)}^{\prime}, \mathscr{F}_{(1)}^{\prime \prime}$ for the new sets of fields. Since every field has been replaced by a field extension, we still get projective systems of fields and, moreover, $G\left(\mathscr{F}_{(1)}^{\prime}\right) \cong G\left(\mathscr{F}^{\prime}\right)$ and $G\left(\mathscr{F}_{(1)}^{\prime \prime}\right) \cong G\left(\mathscr{F}^{\prime \prime}\right)$. Now $\operatorname{atd}\left(F_{(1) i_{1}}^{\prime}\right)=$ $\operatorname{atd}\left(F_{(1) i_{1}}^{\prime \prime}\right)<\infty$, and we proceed by induction.

So, from now on, we assume that our two finite projective systems of fields $\mathscr{F}^{\prime}$ and $\mathscr{F}^{\prime \prime}$ consist of countable fields having the same finite transcendence degree over $\mathbb{Q}$ at each index.

Remark 5.10. Let $K$ be a field equipped with two independent valuations $v_{1}$ and $v_{2}$ and let $\left(L, w_{1}, w_{2}\right)$ be an extension of $\left(K, v_{1}, v_{2}\right)$. Then $w_{1}$ and $w_{2}$ are independent. Indeed, if it were not the case, then $w_{1}$ and $w_{2}$ would define the same topology on $L$ (see [Engler and Prestel 2005, Theorem 2.3.4]), and therefore the same induced topologies on $K$, which coincide with the topologies defined by $v_{1}$ and $v_{2}$. It shows that $v_{1}$ and $v_{2}$ define the same topology on $K$, a contradiction since they are independent (again by the theorem just cited).

Lemma 5.11. There are two henselian valued fields $\left(E_{\perp}^{\prime}, v^{\prime}\right)$ and $\left(E_{\perp}^{\prime \prime}, v^{\prime \prime}\right)$ both containing $\mathbb{Q}(X)$, such that

(1) $v^{\prime}$ and $v^{\prime \prime}$ are of rank one,

(2) $E_{\perp}^{\prime} v^{\prime} \cong F_{\perp}^{\prime}$ and $E_{\perp}^{\prime \prime} v^{\prime \prime} \cong F_{\perp}^{\prime \prime}$,

(3) atd $E_{\perp}^{\prime}=\operatorname{atd} F_{\perp}^{\prime}+1=\operatorname{atd} F_{\perp}^{\prime \prime}+1=\operatorname{atd} E_{\perp}^{\prime \prime}$,

(4) $v^{\prime} E_{\perp}^{\prime}$ and $v^{\prime \prime} E_{\perp}^{\prime \prime}$ are divisible, and

(5) the restrictions of $v^{\prime}$ and $v^{\prime \prime}$ to $\mathbb{Q}(X)$ are independent.

(In (4), two-divisible is actually enough for our purposes.)

Proof. Let $\left\{y_{1}, \ldots, y_{k}\right\}$ be a finite transcendence basis of $F_{\perp}^{\prime}$ over $\mathbb{Q}$, and let $E$ be $\mathbb{Q}\left(y_{1}, \ldots, y_{k}\right)(X)$, equipped with the valuation $v$ determined by the irreducible polynomial $X \in \mathbb{Q}\left(y_{1}, \ldots, y_{k}\right)[X]$. Then $\bar{E} \cong \mathbb{Q}\left(y_{1}, \ldots, y_{k}\right), v E=\mathbb{Z}$ and $F_{\perp}^{\prime}$ is isomorphic to an algebraic extension of $\bar{E}$. By [Endler 1963, Satz 1], there is an algebraic extension $E_{\perp}^{\prime}$ of $E$ and an extension $v^{\prime}$ of $v$ to $E_{\perp}^{\prime}$ such that $E_{\perp}^{\prime}=F_{\perp}^{\prime}$ and $v^{\prime} E_{\perp}^{\prime}$ is divisible of rank one. 
To construct $\left(E_{\perp}^{\prime \prime}, v^{\prime \prime}\right)$, we proceed as above but start with the valuation on $\mathbb{Q}\left(y_{1}, \ldots, y_{k}\right)(X)$ associated to the irreducible polynomial $X-1$. Obviously, $v^{\prime \prime} \uparrow \mathbb{Q}(X)$ and $v^{\prime} \uparrow \mathbb{Q}(X)$ are independent over $\mathbb{Q}(X)$.

We now apply Corollary 2.10 twice (with the valued fields $\left(E_{\perp}^{\prime}, v^{\prime}\right)$ and $\left(E_{\perp}^{\prime \prime}, v^{\prime \prime}\right)$ given by Lemma 5.11), and get two projective systems of henselian valued fields $\mathscr{E}^{\prime}=\left(E_{i}^{\prime}, v_{i}^{\prime}\right)_{i \in I}$ and $\mathscr{E}^{\prime \prime}=\left(E_{i}^{\prime \prime}, v_{i}^{\prime \prime}\right)_{i \in I}$ equipped with valuations of rank one, such that $\operatorname{res}\left(\mathscr{E}^{\prime}\right) \cong \mathscr{F}^{\prime}$ and $\operatorname{res}\left(\mathscr{E}^{\prime \prime}\right) \cong \mathscr{F}^{\prime \prime}$. Up to renaming the transcendental elements, we can assume that for every $i \in I$ there is a finite set $X_{i}$ of transcendental elements over $\mathbb{Q}$ and an algebraic closure $Q_{i}$ of $\mathbb{Q}\left(X_{i}\right)$ such that $E_{i}^{\prime}, E_{i}^{\prime \prime} \subseteq Q_{i}$, and such that, for every $i \leq j \in I \quad X_{i} \subseteq X_{j}$ and $Q_{i} \subseteq Q_{j}$.

Since, for $i \in I, E_{i}^{\prime}$ and $E_{i}^{\prime \prime}$ are both subfields of $Q_{i}$, we can consider the projective system of valued fields $\left(E_{i}^{\prime} \cap E_{i}^{\prime \prime}, v_{i}^{\prime}, v_{i}^{\prime \prime}\right)_{i \in I}$. Note that $v_{i}^{\prime}$ and $v_{i}^{\prime \prime}$ are independent by Remark 5.10 and Lemma 5.11(5). We recall now the following special case of a result from [Heinemann 1985]:

Theorem 5.12. Let $K$ be a field equipped with two independent valuations $v_{1}$ and $v_{2}$. Fix an algebraic closure $\tilde{K}$ of $K$. Let $\left(H_{i}, v_{i}\right)$, for $i=1,2$, be henselian extensions of $\left(K, v_{i}\right)$ such that $H_{1}, H_{2} \subseteq \tilde{K}$ and $K=H_{1} \cap H_{2}$.

Then $\left(H_{i}, v_{i}\right)$ is a henselization of $\left(K, v_{i}\right)$, for $i=1,2$.

Applying this result, we obtain that, for every $i \in I,\left(E_{i}^{\prime}, v_{i}^{\prime}\right)$ is a henselization of $\left(E_{i}^{\prime} \cap E_{i}^{\prime \prime}, v_{i}^{\prime}\right)$ and $\left(E_{i}^{\prime \prime}, v_{i}^{\prime \prime}\right)$ is a henselization of $\left(E_{i}^{\prime} \cap E_{i}^{\prime \prime}, v_{i}^{\prime \prime}\right)$. In particular:

(1) $v^{\prime}\left(E_{i}^{\prime} \cap E_{i}^{\prime \prime}\right)$ and $v^{\prime}\left(E_{i}^{\prime} \cap E_{i}^{\prime \prime}\right)$ are two-divisible;

(2) $\operatorname{res}\left(E_{i}^{\prime} \cap E_{i}^{\prime \prime}, v_{i}^{\prime}\right)_{i \in I} \cong \mathscr{F}^{\prime}$ and $\operatorname{res}\left(E_{i}^{\prime} \cap E_{i}^{\prime \prime}, v_{i}^{\prime \prime}\right)_{i \in I} \cong \mathscr{F}^{\prime \prime}$;

(3) $v^{\prime}$ and $v^{\prime \prime}$ are independent on $E_{i}^{\prime} \cap E_{i}^{\prime \prime}$ (by Lemma 5.11.(5) and Remark 5.10).

We now apply Theorem 5.5 to the system $\left(E_{i}^{\prime} \cap E_{i}^{\prime \prime}, v_{i}^{\prime}, v_{i}^{\prime \prime}\right)_{i \in I}$ and get the system $\left(\mathscr{E}\left(E_{i}^{\prime} \cap E_{i}^{\prime \prime}\right)\right)_{i \in I}$, which satisfies

$$
G\left(\left(\mathscr{E}\left(E_{i}^{\prime} \cap E_{i}^{\prime \prime}\right)\right)_{i \in I}\right) \cong\left(G\left(\left(E_{i}^{\prime} \cap E_{i}^{\prime \prime}\right)^{v_{i}^{\prime}}\right) \times G\left(\left(E_{i}^{\prime} \cap E_{i}^{\prime \prime}\right)^{v_{i}^{\prime \prime}}\right), g_{i j}^{\prime} \times g_{i j}^{\prime \prime}\right)_{i \leq j \in I},
$$

where $g_{i j}^{\prime}$, respectively $g_{i j}^{\prime \prime}$, is the map induced by $\left(E_{i}^{\prime} \cap E_{i}^{\prime \prime}\right)^{v_{i}^{\prime}} \subseteq\left(E_{j}^{\prime} \cap E_{j}^{\prime \prime}\right)^{v_{j}^{\prime}}$, respectively by $\left(E_{i}^{\prime} \cap E_{i}^{\prime \prime}\right)^{v_{i}^{\prime \prime}} \subseteq\left(E_{j}^{\prime} \cap E_{j}^{\prime \prime}\right)^{v_{j}^{\prime \prime}}$. We claim that this last projective system of (Pythagorean) fields is isomorphic to $\left(G\left(F_{i}^{\prime}\right) \times G\left(F_{i}^{\prime \prime}\right), f_{i j}^{\prime} \times f_{i j}^{\prime \prime}\right)_{i \leq j \in I}$. It suffices to check that, for instance, the projective system $\left(G\left(\left(E_{i}^{\prime} \cap E_{i}^{\prime \prime}\right)^{v_{i}^{\prime}}\right), g_{i j}^{\prime}\right)_{i \leq j \in I}$ is isomorphic to $\left(G\left(F_{i}^{\prime}\right), f_{i j}^{\prime}\right)_{i \leq j \in I}$. This is the content of the remainder of this section.

Since $\left(\left(E_{i}^{\prime} \cap E_{i}^{\prime \prime}\right)^{v_{i}^{\prime}}, v_{i}^{\prime}\right)$ is an immediate extension of $\left(E_{i}^{\prime} \cap E_{i}^{\prime \prime}, v_{i}^{\prime}\right)$, we have $\operatorname{res}\left(\left(E_{i}^{\prime} \cap E_{i}^{\prime \prime}\right)^{v_{i}^{\prime}}, v_{i}^{\prime}\right)_{i \in I} \cong \mathscr{F}^{\prime}$, so

$$
G\left(\operatorname{res}\left(\left(E_{i}^{\prime} \cap E_{i}^{\prime \prime}\right)^{v_{i}^{\prime}}, v_{i}^{\prime}\right)_{i \in I}\right) \cong G\left(\mathscr{F}^{\prime}\right)=\left(G\left(F_{i}^{\prime}\right), f_{i j}^{\prime}\right)_{i \leq j \in I}
$$


and it suffices to show that $\left(G\left(\left(E_{i}^{\prime} \cap E_{i}^{\prime \prime}\right)^{v_{i}^{\prime}}, g_{i j}^{\prime}\right)_{i \leq j \in I}\right.$ and $\left.G\left(\operatorname{res}\left(\left(E_{i}^{\prime} \cap E_{i}^{\prime \prime}\right)^{v_{i}^{\prime}}, v_{i}^{\prime}\right)_{i \in I}\right)\right)$ are isomorphic.

We are now in position to conclude by using the following adaptation of the Baer-Krull theorem [Dickmann and Miraglia 2000, Theorem 1.33]. Recall that the functor $G$ is well defined, in general, from the category of unitary commutative rings into the category of $L_{S G}$-structures.

Lemma 5.13. Let $(K, v)$ be a valued field, $i: O_{K} \rightarrow K$ be the inclusion and $q: O_{K} \rightarrow \bar{K}$ be the projection on the quotient $\left(\bar{K}=O_{K} / M_{K}\right)$. Suppose that $v(2)=0$.

(1) The $L_{S G}$-structures $G(K)$ and $G(\bar{K})$ are special groups. The induced $L_{S G^{-}}$ morphism $G(i): G\left(O_{K}\right) \rightarrow G(K)$ is injective and the induced $L_{S G}$-morphism $G(q): G\left(O_{K}\right) \rightarrow G(\bar{K})$ is surjective.

(2) If $(K, v)$ is 2-henselian and $v K=2 v K$, then $G(i): G\left(O_{K}\right) \rightarrow G(K)$ and $G(q): G\left(O_{K}\right) \rightarrow G(\bar{K})$ are $L_{S G}$-isomorphisms. In particular, the $L_{S G^{-}}$ structure $G\left(O_{K}\right)$ is a special group.

(3) If $\left(K^{\prime}, v^{\prime}\right) \supseteq(K, v)$ is a valued field extension, then $O_{K} \subseteq O_{K^{\prime}}, M_{K} \subseteq M_{K^{\prime}}$ and the diagram of special groups below is commutative (where the vertical arrows are induced by the field extension).

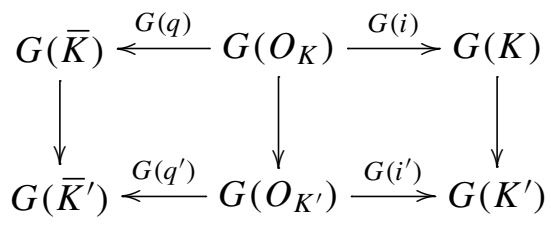

Proof. (1) Since $v(2)=0,2$ is invertible in the rings $K, O_{K}$ and $\bar{K}$, and therefore, as $K$ and $\bar{K}$ are fields, the $L_{S G}$-structures $G(K)$ and $G(\bar{K})$ are special groups ([Dickmann and Miraglia 2000, Theorem 1.32 p.23]). As $q: O_{K} \rightarrow \bar{K}$ is a surjective ring homomorphism, it induces a surjective group homomorphism $\dot{O}_{K} / \dot{O}_{K}^{2} \rightarrow \dot{\bar{K}} / \dot{\bar{K}}^{2}$ and therefore $G(q): G\left(O_{K}\right) \rightarrow G(\bar{K})$ is a surjective $L_{S G^{-}}$ morphism. Now let $a \in \dot{O}_{K}$ such that $a \cdot \dot{K}^{2}=1 . \dot{K}^{2}$; i.e.,there is $b \in \dot{K}$ such that $a=b^{2}$, then $2 v(b)=v(a)=0$ and $b \in \dot{O}_{K}$; therefore $\operatorname{ker}(G(i))=\left\{1 . \dot{O}_{K}^{2}\right\}$ and $G(i): G\left(O_{K}\right) \rightarrow G(K)$ is an injective $L_{S G}$-morphism.

(2) We first prove that $G(q)$ is an $L_{S G}$-isomorphism.

Let $a \in \dot{O}_{K}$ such that $q(a) \cdot \dot{\bar{K}}^{2}=1 . \dot{\bar{K}}^{2}$ then, as $q: O_{K} \rightarrow \bar{K}$ is a surjective ring homomorphism, there is $b \in \dot{O}_{K}$ such that $q(a)=q\left(b^{2}\right)$. Consider now the polynomial $P(t)=t^{2}-a$ in $O_{K}[t]$ : it is a quadratic monic polynomial such that $q(b) \in \bar{K}$ is a root of $P^{q}(t)=t^{2}-q(a)$ in $\bar{K}$ and this root is simple (since $q(a) \neq 0$ and $\operatorname{char}(\bar{K}) \neq 2)$. The hypothesis $(K, v)$ 2-henselian then entails that there is $b^{\prime} \in O_{K}$ such that $q\left(b^{\prime}\right)=q(b)$ and $P\left(b^{\prime}\right)=0$; i.e., $a=b^{\prime 2}$, for some $b^{\prime} \in \dot{O}_{K}$ 
(because $\left.a \in \dot{O}_{K}\right)$. Therefore $\operatorname{ker}(G(q))=\left\{1 . \dot{O}_{K}^{2}\right\}$ and $G(q)$ is an injective $L_{S G^{-}}$ morphism.

We show that whenever $a \in \dot{O}_{K}$, we have

$$
D_{G(\bar{K})}\left\langle 1, G(q)\left(a \dot{O}_{K}^{2}\right)\right\rangle \subseteq G(q)\left[D_{G\left(O_{K}\right)}\left\langle 1, a \dot{O}_{K}^{2}\right\rangle\right] .
$$

Since $q$ and $G(q)$ are surjective, it is enough to prove that for any $z \in \dot{O}_{K}$ such that there are $x, y \in O_{K}$ with $q(z)=1 . q(x)^{2}+q(a) . q(y)^{2}$, there are $z^{\prime} \in \dot{O}_{K}$ and $x^{\prime}, y^{\prime} \in O_{K}$ with $z^{\prime}=1 . x^{\prime 2}+a \cdot y^{\prime 2}$ and

$$
q\left(z^{\prime}\right) \cdot \dot{\bar{K}}^{2}=q(z) \cdot \dot{\bar{K}}^{2}
$$

We recall that $\dot{O}_{K}=O_{K} \backslash M_{K}$ and we split the proof into four cases:

- $x \in M_{K}, y \in M_{K}$ : it is not possible because $q(z) \neq 0$.

- $x \in \dot{O}_{K}, y \in M_{K}$ : then $q(z)=q\left(x^{2}\right)$ and the quadratic monic polynomial $P(t)=t^{2}-z$ over $O_{K}$ has a root in $\bar{K}$, and this root is simple (because $q(z) \neq 0$ and $\operatorname{char}(\bar{K}) \neq 2)$. By the hypothesis that $(K, v)$ is 2-henselian, $P$ has then a root $x^{\prime}$ in $O_{K}$. Taking this $x^{\prime}$ as well as $z^{\prime}:=z$ and $y^{\prime}:=0$ proves the result.

- $x \in M_{K}, y \in \dot{O}_{K}$ : then $q(z)=q\left(a y^{2}\right)$ and the polynomial $P(t)=t^{2}-a^{-1} z$ has a root in $\bar{K}$ and this root is again simple (because $q(z), q(a) \neq 0$ and $\operatorname{char}(\bar{K}) \neq 2$ ). Therefore $P$ has a root $y^{\prime} \in O_{K}$. Taking this $y^{\prime}$ together with $z^{\prime}:=z$ and $x^{\prime}=0$ proves the result.

- $x \in \dot{O}_{K}, y \in \dot{O}_{K}$ : then $q\left((x / y)^{2}+a-z y^{-2}\right)=0$ and the polynomial $P(t)=$ $t^{2}+\left(a-z^{\prime}\right)$, with $z^{\prime}:=z y^{-2}$, is a quadratic monic polynomial in $O_{K}[t]$ such that $q(x / y) \in \bar{K}$ is a root of $P^{q}(t)=t^{2}+q\left(a-z^{\prime}\right)$ in $\bar{K}$ and we may suppose this root is simple (because, if not, as $\operatorname{char}(\bar{K}) \neq 2$, then $q\left(a-z y^{-2}\right)=0$ and we can proceed as in the case just above). Then the hypothesis $(K, v)$ 2-henselian entails that there is $x^{\prime} \in O_{K}$ such that $0=P\left(x^{\prime}\right)=x^{\prime 2}+a-z^{\prime}$ and $q\left(x^{\prime}\right)=q(x / y)$, i.e., such that $z^{\prime}=1 \cdot x^{\prime 2}+a \cdot y^{\prime 2}$, with $y^{\prime}:=1$. Therefore $G(q)\left(z . \dot{O}_{K}^{2}\right)=G(q)\left(z^{\prime} . \dot{O}_{K}^{2}\right) \in G(q)\left[D_{G\left(O_{K}\right)}\left\langle 1, a \dot{O}_{K}^{2}\right\rangle\right]$.

We now prove that $G(i)$ is an $L_{S G}$-isomorphism.

As $v K=2 v K$, for any $a \in \dot{K}$ there is $c \in \dot{K}$ such that $v\left(a c^{2}\right)=0$, i.e., $a c^{2} \in \dot{O}_{K}$. Therefore $G(i)\left(a c^{2} \cdot \dot{O}_{K}^{2}\right)=a \cdot \dot{K}^{2}$ and $G(i)$ is surjective.

To finish the proof, we must check that for each $a \in \dot{O}_{K}$, we have

$$
D_{G(K)}\left\langle 1, G(i)\left(a \dot{O}_{K}^{2}\right)\right\rangle \subseteq G(i)\left[D_{G\left(O_{K}\right)}\left\langle 1, a \dot{O}_{K}^{2}\right\rangle\right] .
$$

Note that if $a=-b^{2}$ for some $b \in \dot{O}_{K}$ then, as $2 \in \dot{O}_{K}$, we have $D_{G\left(O_{K}\right)}\left\langle 1, a \dot{O}_{K}^{2}\right\rangle=$ $\dot{O}_{K} / \dot{O}_{K}^{2}$. Since $G(i)$ is a surjective group homomorphism, we have

$$
G(i)\left[D_{G\left(O_{K}\right)}\left\langle 1, a \dot{O}_{K}^{2}\right\rangle\right]=\dot{\bar{K}} / \dot{\bar{K}}^{2}
$$


and therefore $D_{G(K)}\left\langle 1, G(i)\left(a \dot{O}_{K}^{2}\right)\right\rangle \subseteq G(i)\left[D_{G\left(O_{K}\right)}\left\langle 1, a \dot{O}_{K}^{2}\right\rangle\right]$. Thus we only have to deal with the case $a \notin-\dot{O}_{K}^{2}$ and, again as $G(i)$ is a surjective group homomorphism, it is enough to prove that for any $z \in \dot{O}_{K}$ such that there are $x, y \in K$ with $z=1 \cdot x^{2}+a \cdot y^{2}$, there are $z^{\prime} \in \dot{O}_{K}$ and $x^{\prime}, y^{\prime} \in O_{K}$ such that $z^{\prime}=1 \cdot x^{\prime 2}+a \cdot y^{\prime 2}$ and $z^{\prime} \cdot \dot{K}^{2}=z \cdot \dot{K}^{2}$.

We split the proof into four cases:

- $x, y \in O_{K}$. Then we simply take $z^{\prime}:=z, x^{\prime}:=x$ and $y^{\prime}:=y$.

- $x \in O_{K}$ and $y \notin O_{K}$. Then $y^{-1} \in M_{K}$ and $x / y \in M_{K}$. Thus $(x / y)^{2} \in M_{K}$ and $1(x / y)^{2}+a=z y^{-2} \in M_{K}$. This implies $a \in M_{K}$, a contradiction because $a \in \dot{O}_{K}=O_{K} \backslash M_{K}$.

- $x \notin O_{K}$ and $y=0$. Then $z=x^{2} \notin O_{K}$, a contradiction.

- $x \notin O_{K}$ and $y \neq 0$. Then $z=x^{2}\left(1+a(y / x)^{2}\right) \in \dot{O}_{K}$ and $x^{-1} \in M_{K}$. As $z \in \dot{O}_{K}$, this implies $\left(1+a(y / x)^{2}\right)=z x^{-2} \in M_{K}$, and thus $-a(y / x)^{2} \in 1+M_{K}$. As $(K, v)$ is 2-henselian and $\operatorname{char}(\bar{K}) \neq 2,1+M_{K} \subseteq O_{K}^{2}$ and as $y \neq 0$, then $-a \in K^{2}$. But $-a \in \dot{O}_{K}$, so $-a \in \dot{O}_{K} \cap K^{2}=\dot{O}_{K}^{2}$, contradicting the hypothesis $a \in \dot{O}_{K} \backslash-\dot{O}_{K}^{2}$.

(3) It follows directly from the definition of extension of valued fields that the following diagram of (local) rings and (local) homomorphisms is commutative:

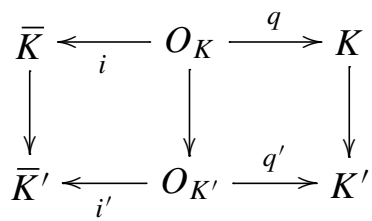

The result follows by applying the functor $G$ to it.

Under the hypotheses of Lemma 5.13, the last item gives us in particular the commutative diagram

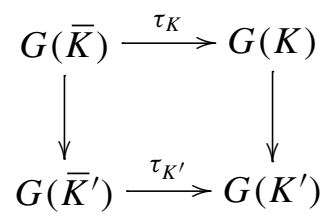

where the maps $\tau_{K}:=G(q) \circ G(i)^{-1}$ and $\tau_{K^{\prime}}:=G\left(q^{\prime}\right) \circ G\left(i^{\prime}\right)^{-1}$ are isomorphisms of special groups whenever $(K, v)$ and $\left(K^{\prime}, v^{\prime}\right)$ are 2-henselian with 2-divisible value groups, and the vertical maps are induced by the field inclusions.

Since, for $i \leq j \in I$, we have an extension of valued fields $\left(\left(E_{i}^{\prime} \cap E_{i}^{\prime \prime}\right)^{v_{i}^{\prime}}, v_{i}^{\prime}\right) \subseteq$ $\left(\left(E_{j}^{\prime} \cap E_{j}^{\prime \prime}\right)^{v_{j}^{\prime}}, v_{j}^{\prime}\right)$ and these two fields are 2-henselian with divisible value groups, 
we conclude that the diagram

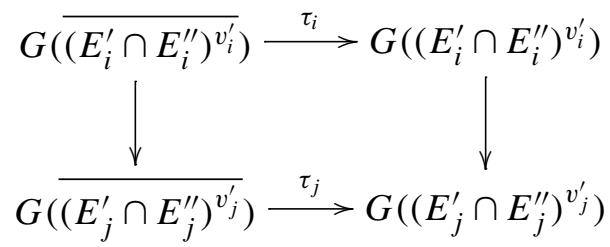

is commutative, where the maps $\tau_{i}$ and $\tau_{j}$ are the isomorphisms corresponding to $\tau_{K}$ and $\tau_{K^{\prime}}$ in (5-2). This concludes the proof of Theorem 5.1.

Gluing, the extension case. Now assume that $G_{\perp} \cong G^{\prime}[H]$, the last case discussed on page 269. Here we have $G_{i} \cong G^{\prime}[H] / \Delta_{i}$ for every $i \in I$, where $H$ is a fixed finite group of exponent $2, \Delta_{i}$ is a saturated subgroup of $G^{\prime}[H]$ and $\Delta_{\perp}=\{1\}$. Furthermore, if $i \leq j \in I$ we have $\Delta_{i} \subseteq \Delta_{j}$ and $f_{i j}$ is naturally identified with the canonical projection from $G^{\prime}[H] / \Delta_{i}$ onto $G^{\prime}[H] / \Delta_{j}$.

In view of this, the following theorem is a reformulation of the last case in the induction step (page 269), and this section is devoted to its proof.

Theorem 5.14. Let $(I, \leq)$ be a finite downward directed index set with first element $\perp$ and last element $T$. Let $G^{\prime}$ be a reduced special group and assume that whenever $\varphi=\left(G_{i}, \eta_{i j}\right)_{i \leq j \in I}$ is a projective system of reduced special groups with $G_{\perp}=G^{\prime}$, then $G$ is realized by a projective system of Pythagorean fields of characteristic zero (where the morphisms are inclusions).

Let $H$ be a finite group of exponent 2 and let $\left(\Delta_{i}\right)_{i \in I}$ be a projective system of saturated subgroups of $G^{\prime}[H]$, where the morphisms are inclusions. Let $\varphi^{\prime}$ be the projective system indexed by I of the special groups $G^{\prime}[H] / \Delta_{i}$, where the morphisms are the canonical projections.

Then ' $G^{\prime}$ is realized by a projective system of Pythagorean fields of characteristic zero (where the morphisms are inclusions).

Notation: If $G$ is a special group and $H$ is a group of exponent 2, we will identify $G$ (respectively $H$ ) with the subgroup $G \times\{1\}$ (respectively $\{1\} \times H$ ) in $G[H]=\{(g, h) \mid g \in G, h \in H\}$ and write $g \cdot h$ for the pair $(g, h)$.

As $H \cong H_{1} \times H_{2}$ entails $G^{\prime}[H] \cong\left(G^{\prime}\left[H_{1}\right]\right)\left[H_{2}\right]$, we may assume $\operatorname{dim}_{\mathbb{F}_{2}} H=1$, i.e., $H=\{1, h\}$ with $h^{2}=1$ and $h \neq 1$.

We define, for $i \in I$ and $i \leq j \in I$ :

$$
\begin{aligned}
& \left.\Omega_{i}:=\Delta_{i} \cap G^{\prime}, \quad G_{i}^{\prime \prime}:=G^{\prime} / \Omega_{i} \text { (note that } \Omega_{i} \subseteq \Omega_{j}\right) \\
& q_{i j}: G_{i}^{\prime \prime} \rightarrow G_{j}^{\prime \prime} \text { the canonical projection, } \\
& \Theta_{i}:=\left\{\left(g \cdot \Omega_{i}\right) . w \in G_{i}^{\prime \prime}[H] \mid g . w \in \Delta_{i}\right\} .
\end{aligned}
$$

The following fact is then easily checked: 
Fact 5.15. (1) $\Omega_{i}$ is a saturated subgroup of $G^{\prime}$.

(2) $\Theta_{i}$ is a saturated subgroup of $G_{i}^{\prime \prime}[H]$ with $G_{i}^{\prime \prime} \cap \Theta_{i}=\{1\}$.

(3) The morphism of special groups $q_{i j} \times \operatorname{Id}: G_{i}^{\prime \prime}[H] \rightarrow G_{j}^{\prime \prime}[H]$ is such that $\left(q_{i j} \times \mathrm{Id}\right)\left(\Theta_{i}\right) \subseteq \Theta_{j}$ and $\left(q_{i j} \times \mathrm{Id}\right)\left\lceil\Theta_{i}: \Theta_{i} \rightarrow \Theta_{j}\right.$ is injective.

(4) The map

$$
\begin{aligned}
\omega_{i}: G^{\prime}[H] / \Delta_{i} & \rightarrow\left(G^{\prime} / \Omega_{i}\right)[H] / \Theta_{i} \\
(g \cdot h) / \Delta_{i} & \mapsto\left(\left(g / \Omega_{i}\right) \cdot h\right) / \Theta_{i}
\end{aligned}
$$

is an isomorphism of special groups.

(5) The diagram

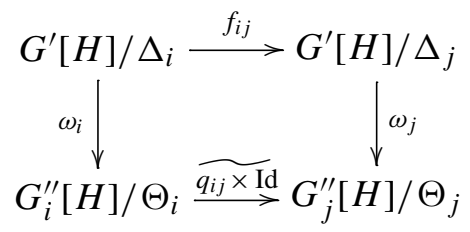

commutes, where $\widetilde{q_{i j} \times \mathrm{I}} \mathrm{d}$ is the canonical map induced on the quotients.

Note that by hypothesis, since $G_{\perp}^{\prime \prime}=G^{\prime}$, the projective system $\left(G_{i}^{\prime \prime}, q_{i j}\right)_{i \leq j \in I}$ is realized by a system of Pythagorean fields $\left(K_{i}\right)_{i \in I}$ of characteristic zero.

To complete the proof, it is then enough to represent the projective system of special groups $\left(G_{i}^{\prime \prime}[H] / \Theta_{i}, \widetilde{q_{i j} \times I} \mathrm{~d}\right)_{i \leq j \in I}$ by some projective system of Pythagorean fields of characteristic zero; this is the content of the following proposition.

Proposition 5.16. There is a projective system of Pythagorean fields of characteristic zero $\left(L_{i}\right)_{i \in I}$, where the morphisms are inclusions, such that

$$
\left(G_{i}^{\prime \prime}[H] / \Theta_{i}, \widetilde{q_{i j} \times \mathrm{I}} \mathrm{d}\right)_{i \leq j \in I} \cong G\left(\left(L_{i}\right)_{i \in I}\right) .
$$

The rest of this section now consists in the proof of Proposition 5.16.

Let us denote by $\gamma_{i j}$ the morphism of special groups induced by $K_{i} \subseteq K_{j}$ :

$$
\left(G\left(K_{i}\right), \gamma_{i j}\right)_{i \leq j \in I} \cong\left(G_{i}^{\prime \prime}, q_{i j}\right)_{i \leq j \in I} .
$$

We define $M_{i}=K_{i}((t))$ for every $i \in I$ and record a well known result:

Lemma 5.17. $\dot{M}_{i} / \dot{M}_{i}{ }^{2}=\left\{a t^{k} \cdot \dot{M}_{i}{ }^{2} \mid a \in \dot{K}_{i}, k \in\{0,1\}\right\}$, and the isomorphism of special groups from $G\left(M_{i}\right)$ to $G\left(K_{i}\right)[H]$ is

$$
\begin{aligned}
\lambda_{i}: G\left(M_{i}\right) & \rightarrow G\left(K_{i}\right)[H] \\
a t^{k} \cdot \dot{M}_{i}{ }^{2} & \mapsto\left(a \cdot \dot{K}_{i}{ }^{2}\right) h^{k} .
\end{aligned}
$$

Proof. This is exactly [Dickmann and Miraglia 2000, Theorem 1.33], where the explicit definition of the isomorphism is given at the beginning of the proof on page 28. 
It immediately follows that

$$
\left(G\left(K_{i}((t))\right), \gamma_{i j} \times \mathrm{Id}\right)_{i \leq j \in I} \cong\left(G_{i}^{\prime \prime}[H], q_{i j} \times \mathrm{Id}\right)_{i \leq j \in I} .
$$

For each $i \in I$, let $\Gamma_{i}$ be the saturated subgroup of $G\left(K_{i}((t))\right)$ that corresponds, by the isomorphisms above, to the saturated subgroup $\Theta_{i}$ of $G_{i}^{\prime \prime}[H]$. This then yields

$$
\left(G\left(K_{i}((t))\right) / \Gamma_{i}, \widetilde{\gamma_{i j} \times \mathrm{I}} \mathrm{d}\right)_{i \leq j \in I} \cong\left(G_{i}^{\prime \prime}[H] / \Theta_{i}, \widetilde{q_{i j} \times \mathrm{Id}}\right)_{i \leq j \in I}
$$

(where $\widetilde{\gamma_{i j} \times \text { Id }}$ denotes the induced map on the quotients), which in turn shows that we only have to find a projective system of fields realizing the system

$$
\left(G\left(K_{i}((t))\right) / \Gamma_{i}, \widetilde{\gamma_{i j} \times \mathrm{Id}}\right)_{i \leq j \in I} .
$$

Therefore, to keep notation simple, we may assume that $G\left(K_{i}\right)[H]=G_{i}^{\prime \prime}[H]$, $\Gamma_{i}=\Theta_{i}, \gamma_{i j} \times \mathrm{Id}=q_{i j} \times \mathrm{Id}$, and that $\widetilde{q_{i j} \times \mathrm{Id}}$ is the map from $G\left(K_{i}\right)[H] / \Theta_{i}$ to $G\left(K_{j}\right)[H] / \Theta_{j}$ induced by $\gamma_{i j} \times \mathrm{Id}=q_{i j} \times \mathrm{Id}$.

In this vein, for every $i \leq j \in I$, we will write $G\left(M_{i}\right)=G_{i}^{\prime \prime}[H], q_{i j} \times \operatorname{Id}$ will stand for the morphism of special groups induced by the inclusion $M_{i} \subseteq M_{j}$, and the diagram

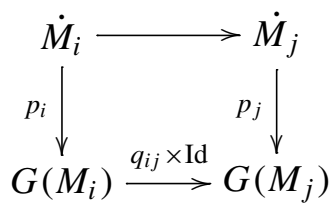

is commutative, where $p_{i}$ and $p_{j}$ denote the canonical maps.

Define $n_{i}:=\operatorname{dim}_{\mathbb{F}_{2}} \Theta_{i}$ for $i \in I$. Note that $\operatorname{dim}_{\mathbb{F}_{2}} \Theta_{i} \leq \operatorname{dim}_{\mathbb{F}_{2}} H=1$, so $n_{i} \in\{0,1\}$. Since $\left(q_{i j} \times \mathrm{Id}\right) \uparrow \Theta_{i}: \Theta_{i} \rightarrow \Theta_{j}$ is injective, we have $n_{i} \leq n_{j}$ whenever $i \leq j \in I$. If $n_{i}=1$, write $\Theta_{i}=\left\{1, a_{i} h\right\}$, with $a_{i} \in G_{i}^{\prime \prime}$. In this case, and if $i \leq j \in I$, we have $\left(q_{i j} \times \mathrm{Id}\right)\left(\Theta_{i}\right)=\Theta_{j}$, so $q_{i j}\left(a_{i}\right)=a_{j}$.

Lemma 5.18. There is $b \in \dot{M}_{\perp}$ such that, for every $i \in I, \Theta_{i} \subseteq\left\{1, p_{i}(b)\right\}$.

Proof. For every $i \leq j \in I$, the map $q_{i j}$ is surjective. In particular the map $q_{\perp \top}$ is surjective and, by diagram (5-4) above, $p_{\top}\left(\dot{M}_{\perp}\right)=\operatorname{Im}\left(q_{\perp \top} \times \operatorname{Id}\right)=G\left(M_{\top}\right)$. Let $b \in \dot{M}_{\perp}$ be such that $\left\{1, p_{\top}(b)\right\}=\Theta_{\top}$. Let now $i \in I$ and let $x \in \Theta_{i}$. Then

$$
\left(q_{i \top} \times \mathrm{Id}\right)(x) \in \Theta_{\top}=\left\{1, p_{\top}(b)\right\} .
$$

If $\left(q_{i \top} \times \mathrm{Id}\right)(x)=1$, we get $x=1 \in\left\{1, p_{i}(b)\right\}$, because $\left(q_{i j} \times \mathrm{Id}\right)\left\lceil: \Theta_{i} \rightarrow \Theta_{j}\right.$ is an injective group homomorphism. If $\left(q_{i \top} \times \mathrm{Id}\right)(x)=p_{\top}(b)$, since diagram (5-4) is commutative, we get $p_{\top}(b)=\left(q_{i} \top \times \mathrm{Id}\right)\left(p_{i}(b)\right)$, so $\left(q_{i} \top \times \mathrm{Id}\right)(x)=\left(q_{i \top} \times \mathrm{Id}\right)\left(p_{i}(b)\right)$ and we conclude that $x=p_{i}(b)$. 
We assume from now on that there is $i \in I$ such that $n_{i}=1$ (equivalently, $n_{\top}=1$ ). Otherwise $\Theta_{j}=\{1\}$ for every $j \in I$, and the projective system of fields $\left(M_{i}\right)_{i \in I}$ realizes the projective system of special groups $\left(G_{i}^{\prime \prime}[H] / \Theta_{i}, \widetilde{q_{i j} \times I} \mathrm{~d}\right)_{i \leq j \in I}$.

Recall that an element $a$ of a special group $T$ is called rigid when $a \neq 1$ and $D_{T}\langle 1, a\rangle=\{1, a\}$ and an element $b$ of $T$ is birigid when $b$ and $-b$ are rigid. If $T=G[H]$, then every element in $G[H] \backslash G$ is birigid (this is essentially the only way to obtain birigid elements in a special group; see [Dickmann and Miraglia 2000, p. 12, Berman's Theorem]).

Since we assume that $n_{i}=1$ for some $i \in I$ (in other words $n_{\top}=1$ ), it follows that the element $b$ produced in Lemma 5.18 is birigid in $M_{i}$ for every $i \in I$.

The next proposition uses the following notation: If $K$ is a field and $a \in K$ then $K(\sqrt[\infty]{a})$ stands for $K\left(\sqrt[2^{n}]{a}, n \in \mathbb{N}\right)$.

Proposition 5.19 [Becher 2002, Proposition 8.2]. Let $F$ be a field, let a be a birigid element in $F$ (i.e., $a \in \dot{F}$ and a. $\dot{F}^{2}$ is birigid in $G(F)$ ) and let $\varphi$ be a quadratic form over $F$. Let $L:=F(\sqrt[\infty]{a})$. Then

(1) $\dot{L}=\dot{F} \dot{L}^{2}$ and $\dot{F} \cap \dot{L}^{2}=\dot{F}^{2} \cup a \dot{F}^{2}$;

(2) $\varphi$ is isotropic over $L$ if and only if $\varphi \oplus a \varphi$ is isotropic over $F$.

We define, for $i \in I$,

$$
L_{i}= \begin{cases}M_{i} & \text { if } n_{i}=0 \\ M_{i}(\sqrt[\infty]{b}) & \text { if } n_{i}=1\end{cases}
$$

Since $n_{i}=1$ implies $n_{j}=1$ whenever $i \leq j \in I$, the system $\left(L_{i}\right)_{i \in I}$ is a projective system of fields. Note that the following diagram of fields is obviously commutative (with the natural inclusions as morphisms):

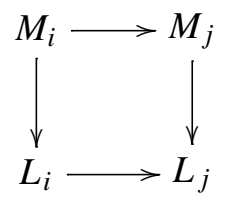

which implies that the induced diagram of special groups is also commutative:

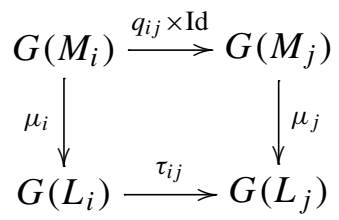

where $\mu_{i}: G\left(M_{i}\right) \rightarrow G\left(L_{i}\right)$ is the map induced by $M_{i} \subseteq L_{i}$ and $\tau_{i j}$ is the map induced by $L_{i} \subseteq L_{j}$. 
Lemma 5.20. For $i \in I$, let $\pi_{i}: G\left(K_{i}\right)[H] \rightarrow G\left(K_{i}\right)[H] / \Theta_{i}$ be the canonical projection. Then $\mu_{i}$ is surjective and there is a unique isomorphism of special groups $\xi_{i}: G\left(L_{i}\right) \rightarrow G\left(K_{i}\right)[H] / \Theta_{i}$ such that the diagram

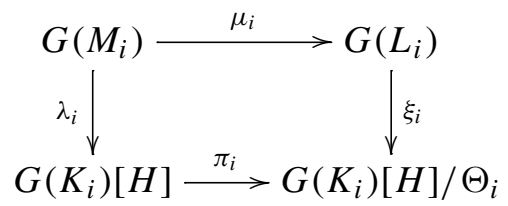

is commutative. In particular, $L_{i}$ is Pythagorean.

Proof. The case $n_{i}=0$ is trivial, so we assume $n_{i}=1$. To avoid unnecessary notational complications, if $K$ is a field and $x \in \dot{K}$, we simply write $\bar{x}$ for the class of $x$ in $\dot{K} / \dot{K}^{2}$. By Proposition 5.19(1) we know that $\mu_{i}$ is surjective and that $\operatorname{ker}\left(\mu_{i} \circ \lambda_{i}^{-1}\right)=\left\{1, \lambda_{i}(\bar{b})\right\}=D_{G\left(K_{i}\right)[H]}\left\langle 1, \lambda_{i}(\bar{b})\right\rangle$. In particular, there is a unique isomorphism of groups $\xi_{i}: G\left(L_{i}\right) \rightarrow G\left(K_{i}\right)[H] /\left\{1, \lambda_{i}(\bar{b})\right\}$ such that the following diagram commutes:

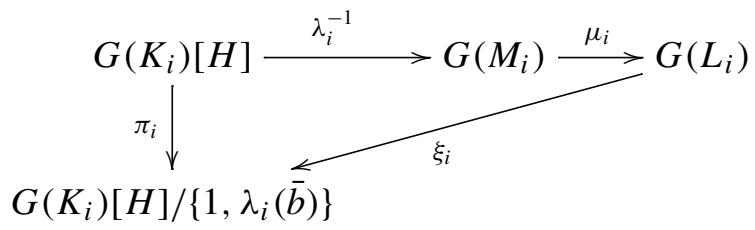

We show that $\xi_{i}$ is an isomorphism of special groups. The image of -1 is clearly -1 . Take $\mu_{i}(\bar{c}), \mu_{i}(\bar{d}) \in G\left(L_{i}\right)$, where $c, d \in \dot{M}_{i}$. We have

$$
\begin{aligned}
\mu_{i}(\bar{c}) \in D_{G\left(L_{i}\right)}\left\langle 1, \mu_{i}(\bar{d})\right\rangle & \Leftrightarrow c \in D_{L_{i}}\langle 1, d\rangle \\
& \Leftrightarrow\langle\langle-c, d\rangle\rangle \text { isotropic over } L_{i} \\
& \Leftrightarrow\langle\langle-c, d\rangle\rangle \oplus b\langle\langle-c, d\rangle\rangle \text { isotropic over } M_{i},
\end{aligned}
$$

the last equivalence following from Proposition 5.19(2). Recalling that Pfister forms are isotropic if and only if they are hyperbolic, we continue the chain of equivalences with

$\Leftrightarrow\langle 1, b\rangle \otimes\langle\langle-c, d\rangle\rangle$ isotropic over $M_{i}$

$\Leftrightarrow\langle 1, b\rangle \otimes\langle\langle-c, d\rangle\rangle$ hyperbolic over $M_{i}$

$\Leftrightarrow\left\langle 1, \lambda_{i}(\bar{b})\right\rangle \otimes\left\langle\left\langle-\lambda_{i}(\bar{c}), \lambda_{i}(\bar{d})\right\rangle\right\rangle$ hyperbolic in $G\left(K_{i}\right)[H]$

$\Leftrightarrow\left\langle 1, \lambda_{i}(\bar{b})\right\rangle \otimes\left\langle\left\langle-\lambda_{i}(\bar{c}), \lambda_{i}(\bar{d})\right\rangle\right\rangle \equiv\left\langle 1, \lambda_{i}(\bar{b})\right\rangle \otimes\langle-1,1,-1,1\rangle$ in $G\left(K_{i}\right)[H]$

$\Leftrightarrow\left\langle\left\langle-\pi_{i} \circ \lambda_{i}(\bar{c}), \pi_{i} \circ \lambda_{i}(\bar{d})\right\rangle\right\rangle \equiv\langle-1,1,-1,1\rangle$

$$
\text { in } G\left(K_{i}\right)[H] / D\left\langle 1, \lambda_{i}(\bar{b})\right\rangle=G\left(K_{i}\right)[H] /\left\{1, \lambda_{i}(\bar{b})\right\},
$$

the last step following from [Dickmann and Miraglia 2000, Proposition 2.21]. But this last condition is equivalent to $\left\langle\left\langle-\pi_{i} \circ \lambda_{i}(\bar{c}), \pi_{i} \circ \lambda_{i}(\bar{d})\right\rangle\right\rangle$ being hyperbolic in 
$G\left(K_{i}\right)[H] /\left\{1, \lambda_{i}(\bar{b})\right\}$, and so to $\pi_{i} \circ \lambda_{i}(\bar{c})$ lying in $D_{G\left(K_{i}\right)[H] /\left\{1, \lambda_{i}(\bar{b})\right\}}\left\langle 1, \pi_{i} \circ \lambda_{i}(\bar{d})\right\rangle$. This shows that $\xi_{i}$ is an isomorphism of special groups. Since $\lambda_{i}(b)$ is birigid and $|H|=2$, we obtain that $G\left(L_{i}\right)$, being isomorphic to $G\left(K_{i}\right)[H] /\left\{1, \lambda_{i}(\bar{b})\right\} \cong G\left(K_{i}\right)$, is a reduced special group or $\{1\}$, which entails that $L_{i}$ is a Pythagorean field.

Recall that, using the identifications made after (5-3), we have

$$
\left(\widetilde{q_{i j} \times \mathrm{Id}}\right)\left(\left(a \dot{K}_{i}^{2}\right) h^{k} \cdot \Theta_{i}\right)=\left(a \dot{K}_{j}^{2}\right) h^{k} \cdot \Theta_{j} \quad \text { for } a \in \dot{K}_{i} \text { and } k \in\{0,1\} .
$$

Proposition 5.21. The diagram

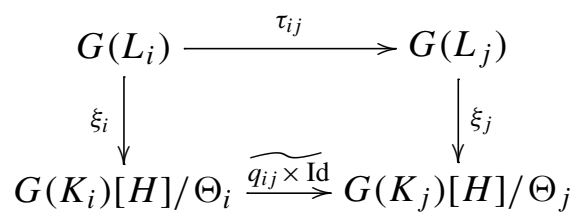

commutes. In particular, $G\left(\left(L_{i}\right)_{i \in I}\right) \cong\left(G_{i}^{\prime \prime}[H] / \Theta_{i}, \widetilde{q_{i j} \times \mathrm{I}} \mathrm{d}\right)_{i \leq j \in I}$ and

$$
\lim _{\longleftarrow} G\left(\left(L_{i}\right)_{i \in I}\right) \cong \lim \left(G_{i}, f_{i j}\right)_{i \leq j \in I} .
$$

Proof. Since $\mu_{i}$ and $\mu_{j}$ are surjective by Lemma 5.20, the commutative diagram in that same lemma completely determines $\xi_{i}$ and $\xi_{j}$. Let $z=\mu_{i}\left(a t^{k} \dot{M}_{i}{ }^{2}\right) \in G\left(L_{i}\right)$ (with $a \in \dot{K}_{i}$ and $k \in\{0,1\}$ ). Then

(5-6) $\xi_{i}(z)=\xi_{i} \circ \mu_{i}\left(a t^{k} \dot{M}_{i}{ }^{2}\right)=\pi_{i} \circ \lambda_{i}\left(a t^{k} \dot{M}_{i}{ }^{2}\right)=\pi_{i}\left(\left(a \dot{K}_{i}{ }^{2}\right) h^{k}\right)=\left(\left(a \dot{K}_{i}{ }^{2}\right) h^{k}\right) \cdot \Theta_{i}$.

where the second equality comes from Lemma 5.20. Applying this, we obtain $\left(\widetilde{q_{i j} \times \mathrm{I}} \mathrm{d}\right) \circ \xi_{i}(z)=\left(\widetilde{q_{i j} \times \mathrm{I}} \mathrm{d}\right)\left(\left(\left(a \dot{K}_{i}^{2}\right) h^{k}\right) \cdot \Theta_{i}\right)=\left(a \dot{K}_{j}{ }^{2}\right) h^{k} \cdot \Theta_{j}$ and

$$
\begin{aligned}
\xi_{j} \circ \tau_{i j}(z) & =\xi_{j} \circ \tau_{i j} \circ \mu_{i}\left(a t^{k} \dot{M}_{i}{ }^{2}\right) & & \\
& =\xi_{j} \circ \mu_{j} \circ\left(q_{i j} \times \mathrm{Id}\right)\left(a t^{k} \dot{M}_{i}{ }^{2}\right) & & \text { by diagram (5-5) } \\
& =\xi_{j} \circ \mu_{j}\left(a t^{k} \dot{M}_{j}{ }^{2}\right) & & \text { since } q_{i j} \times \text { Id is induced by } M_{i} \subseteq M_{j} \\
& =\left(a \dot{K}_{j}{ }^{2}\right) h^{k} \cdot \Theta_{j} & & \text { by (5-6), }
\end{aligned}
$$

which finishes the proof.

\section{Acknowledgement}

Both authors wish to thank the referee for many detailed and helpful comments.

\section{References}

[Astier and Tressl 2005] V. Astier and M. Tressl, "Axiomatization of local-global principles for pp-formulas in spaces of orderings", Arch. Math. Logic 44:1 (2005), 77-95. MR 2005k:03088 Zbl 1099.03027 
[Becher 2002] K. J. Becher, "Le radical de Kaplansky", Publ. Mat. de Besançon, Univ. FrancheComté, 2002, available at http://www.math.uni-konstanz.de/ becher/art/kap.pdf. MR 2004g:11030 Zbl 1161.11328

[Bröcker 1977] L. Bröcker, "Über die Anzahl der Anordnungen eines kommutativen Körpers", Arch. Math. (Basel) 29:5 (1977), 458-464. MR 58 \#5613

[Chang and Keisler 1990] C. C. Chang and H. J. Keisler, Model theory, 3rd ed., Studies in Logic and the Foundations of Math. 73, North-Holland, Amsterdam, 1990. MR 91c:03026 Zbl 0697.03022

[Dickmann and Miraglia 2000] M. A. Dickmann and F. Miraglia, Special groups: Boolean-theoretic methods in the theory of quadratic forms, Mem. Amer. Math. Soc. 689, Amer. Math. Soc., Providence, RI, 2000. MR 2000j:11052 Zbl 1052.11027

[Dickmann and Miraglia 2003] M. A. Dickmann and F. Miraglia, "Bounds for the representation of quadratic forms”, J. Algebra 268:1 (2003), 209-251. MR 2004k:11052 Zbl 1053.11035

[Endler 1963] O. Endler, "Über einen Existenzsatz der Bewertungstheorie", Math. Ann. 150 (1963), 54-65. MR 26 \#3732 Zbl 0142.28302

[Engler and Prestel 2005] A. J. Engler and A. Prestel, Valued fields, Springer, Berlin, 2005. MR 2007a:12005 Zbl 1128.12009

[Heinemann 1985] B. Heinemann, "On finite intersections of "Henselian valued" fields", Мапиscripta Math. 52:1-3 (1985), 37-61. MR 87a:12013

[Hodges 1993] W. Hodges, Model theory, Encyclopedia of Mathematics and its Applications 42, Cambridge University Press, Cambridge, 1993. MR 94e:03002 Zbl 0789.03031

[Kula 1979] M. Kula, "Fields with prescribed quadratic form schemes", Math. Z. 167:3 (1979), 201-212. MR 80f:10024 Zbl 0388.10017

[Kula et al. 1984] M. Kula, M. A. Marshall, and A. Sładek, "Direct limits of finite spaces of orderings", Pacific J. Math. 112:2 (1984), 391-406. MR 85f:11023 Zbl 0535.10020

[Lira de Lima 1997] A. Lira de Lima, Les groupes spéciaux: aspects algébriques et combinatoires de la théorie des espaces d'ordres abstraits, Ph.D. thesis, Université de Paris 7, Paris, 1997.

[Mariano 2003] H. L. Mariano, Contribuições à teoria dos grupos especiais, Ph.D. thesis, Universidade de São Paulo, São Paulo, 2003.

[Marshall 1980] M. Marshall, "Spaces of orderings, IV", Canad. J. Math. 32:3 (1980), 603-627. MR 81m:10035 Zbl 0433.10009

Received February 1, 2010. Revised September 7, 2010.

VINCENT ASTIER

SCHOOL OF MATHEMATICAL SCIENCES

University COLLEGE DUbLin

BELFIELD, DUBLIN 4

IRELAND

vincent.astier@ucd.ie

HUGO MARIANO

Instituto de Matemática E Estatística da Universidade de São PaUlo

RUA DO MATÃo, 1010 (CIDAdE UNIVERSITÁRIA)

05508-090 SÃo PAULO

BRAZIL

hugomar@ime.usp.br 


\title{
PACIFIC JOURNAL OF MATHEMATICS
}

\author{
http://www.pjmath.org \\ Founded in 1951 by
}

E. F. Beckenbach (1906-1982) and F. Wolf (1904-1989)

\section{EDITORS}

V. S. Varadarajan (Managing Editor)

Department of Mathematics

University of California

Los Angeles, CA 90095-1555

pacific@math.ucla.edu

Vyjayanthi Chari

Department of Mathematics

University of California

Riverside, CA 92521-0135

chari@math.ucr.edu

\section{Robert Finn}

Department of Mathematics Stanford University

Stanford, CA 94305-2125

finn@math.stanford.edu

Kefeng Liu

Department of Mathematics

University of California

Los Angeles, CA 90095-1555

liu@math.ucla.edu
Darren Long

Department of Mathematics

University of California

Santa Barbara, CA 93106-3080

long@math.ucsb.edu

Jiang-Hua Lu

Department of Mathematics

The University of Hong Kong

Pokfulam Rd., Hong Kong jhlu@maths.hku.hk

Alexander Merkurjev

Department of Mathematics University of California

Los Angeles, CA 90095-1555 merkurev@math.ucla.edu
Sorin Popa

Department of Mathematics

University of California

Los Angeles, CA 90095-1555

popa@math.ucla.edu

Jie Qing

Department of Mathematics

University of California

Santa Cruz, CA 95064

qing@ cats.ucsc.edu

Jonathan Rogawski

Department of Mathematics

University of California

Los Angeles, CA 90095-1555

jonr@math.ucla.edu

\section{PRODUCTION}

pacific@math.berkeley.edu

Silvio Levy, Scientific Editor Matthew Cargo, Senior Production Editor

ACADEMIA SINICA, TAIPEI

CALIFORNIA INST. OF TECHNOLOGY

INST. DE MATEMÁTICA PURA E APLICADA

KEIO UNIVERSITY

MATH. SCIENCES RESEARCH INSTITUTE

NEW MEXICO STATE UNIV.

OREGON STATE UNIV.

\section{SUPPORTING INSTITUTIONS}

STANFORD UNIVERSITY
UNIV. OF BRITISH COLUMBIA
UNIV. OF CALIFORNIA, BERKELEY
UNIV. OF CALIFORNIA, DAVIS
UNIV. OF CALIFORNIA, LOS ANGELES
UNIV. OF CALIFORNIA, RIVERSIDE
UNIV. OF CALIFORNIA, SAN DIEGO
UNIV. OF CALIF., SANTA BARBARA

UNIV. OF CALIF., SANTA CRUZ

UNIV. OF MONTANA

UNIV. OF OREGON

UNIV. OF SOUTHERN CALIFORNIA

UNIV. OF UTAH

UNIV. OF WASHINGTON

WASHINGTON STATE UNIVERSITY

These supporting institutions contribute to the cost of publication of this Journal, but they are not owners or publishers and have no responsibility for its contents or policies.

See inside back cover or www.pjmath.org for submission instructions.

The subscription price for 2011 is US \$420/year for the electronic version, and \$485/year for print and electronic.

Subscriptions, requests for back issues from the last three years and changes of subscribers address should be sent to Pacific Journal of Mathematics, P.O. Box 4163, Berkeley, CA 94704-0163, U.S.A. Prior back issues are obtainable from Periodicals Service Company, 11 Main Street, Germantown, NY 12526-5635. The Pacific Journal of Mathematics is indexed by Mathematical Reviews, Zentralblatt MATH, PASCAL CNRS Index, Referativnyi Zhurnal, Current Mathematical Publications and the Science Citation Index.

The Pacific Journal of Mathematics (ISSN 0030-8730) at the University of California, c/o Department of Mathematics, 969 Evans Hall, Berkeley, CA 94720-3840, is published monthly except July and August. Periodical rate postage paid at Berkeley, CA 94704, and additional mailing offices. POSTMASTER: send address changes to Pacific Journal of Mathematics, P.O. Box 4163, Berkeley, CA 94704-0163.

PJM peer review and production are managed by EditFLOW ${ }^{\mathrm{TM}}$ from Mathematical Sciences Publishers.

PUBLISHED BY PACIFIC JOURNAL OF MATHEMATICS

at the University of California, Berkeley 94720-3840

A NON-PROFIT CORPORATION

Typeset in IATEX

Copyright $(2011$ by Pacific Journal of Mathematics 


\section{PACIFIC JOURNAL OF MATHEMATICS}

Volume $250 \quad$ No. $2 \quad$ April 2011

Realizing profinite reduced special groups

VinCENT Astier and Hugo Mariano

On fibered commensurability

DANNy CALEGARI, Hongbin SUN and Shicheng WANG

On an overdetermined elliptic problem

LAURENT HaUswirTh, FrÉdÉRIC HÉLEIN and FranK PACARD

Minimal sets of a recurrent discrete flow

HATTAB HAWETE

Trace-positive polynomials

IGOR KLEP

Remarks on the product of harmonic forms

LiviU ORnea and Mihaela Pilca

Steinberg representation of GSp(4): Bessel models and integral representation of $L$-functions

Ameya Pitale

An integral expression of the first nontrivial one-cocycle of the space of long knots in $\mathbb{R}^{3}$

KEIICHI SAKAI

Burghelea-Haller analytic torsion for twisted de Rham complexes

GUANGXIANG SU

$K(n)$-localization of the $K(n+1)$-local $E_{n+1}$-Adams spectral sequences

TAKESHI TORII

Thompson's group is distorted in the Thompson-Stein groups

Claire Wladis

Parabolic meromorphic functions 